In press at The Astrophysical Journal

Preprint typeset using LATEX style emulateapj v. 08/22/09

\title{
IDENTIFYING ANOMALIES IN GRAVITATIONAL LENS TIME DELAYS
}

\author{
Arthur B. Congdon ${ }^{1,2}$, Charles R. Keeton ${ }^{2}$, and C. Erik Nordgren ${ }^{3}$ \\ In press at The Astrophysical Journal
}

\begin{abstract}
We examine the ability of gravitational lens time delays to reveal complex structure in lens potentials. In a previous paper, we predicted how the time delay between the bright pair of images in a "fold" lens scales with the image separation, for smooth lens potentials. Here we show that the proportionality constant increases with the quadrupole moment of the lens potential, and depends only weakly on the position of the source along the caustic. We use Monte Carlo simulations to determine the range of time delays that can be produced by realistic smooth lens models consisting of isothermal ellipsoid galaxies with tidal shear. We can then identify outliers as "time delay anomalies." We find evidence for anomalies in close image pairs in the cusp lenses RX J1131-1231 and B1422+231. The anomalies in RX J1131-1231 provide strong evidence for substructure in the lens potential, while at this point the apparent anomalies in B1422+231 mainly indicate that the time delay measurements need to be improved. We also find evidence for time delay anomalies in larger-separation image pairs in the fold lenses, B1608+656 and WFI 2033-4723, and the cusp lens RX J0911+0551. We suggest that these anomalies are caused by some combination of substructure and a complex lens environment. Finally, to assist future monitoring campaigns we use our smooth models with shear to predict the time delays
\end{abstract} for all known four-image lenses.

Subject headings: gravitational lensing - cosmology: dark matter - cosmology: theory - galaxies: structure - methods: numerical

\section{INTRODUCTION}

Gravitational lensing has become a valuable probe of dark matter substructure in distant galaxies (see $\S \mathrm{B} .8$ of Kochanek et al. 2006b, and references therein). A growing body of evidence suggests that anomalous flux ratios, which are observed in many four-image quasar lenses (Metcalf \& Zhao 2002; Keeton et al. 2003, 2005), can be explained if a few percent of the projected mass within each lens galaxy's Einstein angle (which sets the spatial scale for lensing) is contained in cold dark matter (CDM) "clumps" (Mao \& Schneider 1998; Metcalf \& Madau 2001; Chiba 2002; Dalal \& Kochanek 2002; Kochanek \& Dalal 2004). It is then natural to ask whether it is possible to use lensing to measure properties beyond the mean substructure mass fraction. Of particular interest is the clump mass function, because the discrepancy between the observed number of Local Group satellite galaxies and the theoretically predicted abundance of dark matter clumps varies strongly with mass (e.g., Klypin et al. 1999; Moore et al. 1999; Strigari et al. 2007). Unfortunately, it is difficult to constrain the mass function with anomalous flux ratios, because there is a degeneracy such that a small clump near a lensed image (in projected distance) can produce the same flux perturbation as a large clump farther away (see Dalal \& Kochanek 2002).

One possible solution to this problem is to measure

1 Jet Propulsion Laboratory, California Institute of Technology, 4800 Oak Grove Drive, MS 169-327, Pasadena, CA 91109-8001; acongdon@jpl.nasa.gov

2 Department of Physics and Astronomy, Rutgers University, 136 Frelinghuysen Road, Piscataway, NJ 08854-8019; keeton@physics.rutgers.edu

3 Department of Chemistry, University of Pennsylvania, 231 South 34th Street, Philadelphia, PA 19104-6323; nordgren@sas.upenn.edu flux ratios at multiple wavelengths. Quasar emission regions are believed to have different sizes at different wavelengths. Roughly speaking, only clumps with Einstein radii (Einstein angles translated into units of length) larger than the size of the source can produce flux ratio anomalies (e.g., Dobler \& Keeton 2006); clumps with small Einstein radii act collectively as a smooth mass component. Since the Einstein radius depends on mass, the source size effectively translates into a mass threshold. The net effect is that multi-wavelength observations provide a way to study clumps with different mass ranges. ${ }^{4}$ For example, continuum (Woźniak et al. 2000) and broad-line (Richards et al. 2004; Keeton et al. 2006) optical emission regions in quasars can be perturbed by objects of stellar mass and larger. Radio emission regions, by contrast, are large enough that only objects with masses $\gtrsim 10^{6} M_{\odot}$ are important (Dobler \& Keeton 2006). If a flux-ratio anomaly is observed at optical wavelengths but not at radio wavelengths, it is likely that microlensing by stars in the lens galaxy is the culprit. To avoid contamination by microlensing, Dalal \& Kochanek (2002) focused on radio anomalies in their study. Infrared emission regions are intermediate in size, so observations in this band provide a way to probe objects with mass scales between stars and clumps. Recent mid-IR observations with the Spitzer (Poindexter et al. 2007a, b) and Subaru (Chiba et al. 2005; Minezaki et al. 2009) telescopes have achieved the spatial resolution necessary for strong lensing studies. In particular, Chiba et al. (2005) found evidence for subhalos of $\sim 10^{4} M_{\odot}$ in the lens system B1422+231, and possibly in PG $1115+080$ as well. In addition, Minezaki et al. (2009) recently found evidence of

\footnotetext{
4 This is similar to the idea of "chromatic" microlensing, which is discussed by Kochanek et al. (2007).
} 
a clump with mass $>10^{5} M_{\odot}$ in the lens MG $0414+0534$. A related way to study the substructure mass function is to compare continuum and broad-line emission in optical lenses (Moustakas \& Metcalf 2003), which originate from the central accretion disk and an extended distribution of fast-moving clouds, respectively. These two regions differ in length scale by a factor of $\sim 100$, whence their utility for substructure lensing. This technique has been employed to study the four-image lenses HE 0435-1223 (Wisotzki et al. 2003), SDSS J0924+0219 (Keeton et al. 2006; Eigenbrod et al. 2006), SDSS J1004+4112 (Richards et al. 2004), RX J1131-1231 (Sluse et al. 2007), and Q2237+0305 (Metcalf et al. 2004; Eigenbrod et al. 2008), along with several twoimage lenses (Burud et al. 2002; Inada et al.|2005a, 2006; Sluse et al. 2008)

While the method of probing substructure with differential source size effects is promising, this approach does have some limitations. For one thing, the mass threshold defined by the source size and Einstein radius does not represent a sharp cutoff (see Dobler \& Keeton 2006), so it is difficult to obtain tight constraints on the clump mass. In addition, it may not be possible to measure flux ratios at enough wavelengths to sample the mass function well. Finally, lensing constraints on the mass function will only be as accurate as the mapping of wavelength to source size. While the model of Shakura \& Sunvaev (1973) has been quite successful in explaining the observed properties of accretion disks, and gives a relation between wavelength and source size, it does not apply to the full range of wavelengths relevant to strong lensing.

A second possible approach to constrain the substructure mass function is to use flux ratios in combination with other lens observables. Although they are smaller in amplitude than flux ratio perturbations, substructure effects on both image positions (Chen et al. 2007) and time delays (Keeton \& Moustakas 2009) should be detectable. Combining different observables will be valuable because they depend on the lens potential in different ways: time delays, image positions, and flux ratios depend on the potential and its first and second derivatives, respectively. The different observables, in other words, contain different information about the substructure population. For example, Keeton \& Moustakas (2009) showed that time delays are sensitive to the slope and dynamic range of the substructure mass function, which suggests that precise time delay measurements may provide a way to constrain these properties. Before we can use this method, we need to determine whether observed time delays differ from the predictions for smooth mass models in a way that may indicate the presence of substructure. Developing a method to identify such "time delay anomalies" is the focus of this paper.

In particular, we wish to understand the range of time delays that can be produced by reasonable smooth models. We can then classify any outlier as "anomalous" and use it as evidence of complexity in the lens potential. We must be careful when interpreting anomalies, however, because dark matter substructure may not be the only relevant source of complexity in the lens galaxy. Stars also constitute complex structure that is important when interpreting flux ratios (due to microlensing), but they have essentially no effect on time delays (Keeton \& Moustakas 2009). This means that time de- lays should not depend on wavelength, so we do not need to distinguish between radio and optical time delays. ${ }^{5} \mathrm{Fi}-$ nally, extinction by dust in the lens galaxy can perturb flux ratios, but it will not have any effect on time delays, which are measured through flux variability alone and do not depend on color information.

The other main source of complexity we need to consider is the environment of the lens galaxy. Many lens galaxies lie in groups or clusters of galaxies (e.g., Momcheva et al. 2006; Auger et al. 2007). To lowest order, such an environment contributes a tidal shear to the lens potential (e.g., Keeton et al. 1997), and we therefore include shear in our analysis, but there may be higher-order terms that are non-negligible. Extreme examples of this situation include the lens B1608+656, which has two galaxies inside the Einstein angle (Koopmans \& Fassnacht 1999; Koopmans et al. 2003; Suvu et al. 2009), and the lens SDSS J1004+4112, which is produced by a cluster of galaxies (Inada et al. 2003; Oguri et al. 2004). In general, time delay anomalies in close pairs of images potentially provide the strongest evidence of dark matter substructure, because environmental effects are fairly large-scale and should not produce dramatic differences between images separated by a distance much less than the Einstein angle. With time delay anomalies in image pairs that have larger separations, by contrast, we will need to take more care to consider environmental effects as well as substructure.

Our approach follows that of Keeton et al. (2003, 2005), who employed analytic flux-ratio relations that are generic for all lenses with fold and cusp configurations produced by smooth mass models. To be more specific, a fold lens contains a bright pair of images whose fluxes $F_{A}$ and $F_{B}$ should satisfy the relation

$$
R_{\text {fold }} \equiv \frac{F_{A}-F_{B}}{F_{A}+F_{B}} \approx A_{\text {fold }} d_{1},
$$

where $d_{1}$ is the image separation and $A_{\text {fold }}$ depends on properties of the lens potential. A cusp lens contains a triplet of bright images whose fluxes should satisfy the relation

$$
R_{\text {cusp }} \equiv \frac{F_{A}-F_{B}+F_{C}}{F_{A}+F_{B}+F_{C}} \approx A_{\text {cusp }} d_{1}^{2},
$$

where $d_{1}$ is the distance between the closest pair of images and $A_{\text {cusp }}$ depends on properties of the lens potential (see, e.g., Congdon et al. 2008). (Note that $R_{\text {fold }}$ and $R_{\text {cusp }}$ vanish in the limit $d_{1} \rightarrow 0$.) If these relations are violated, we may conclude that the mass distribution of the lens galaxy cannot be smooth and must contain additional structure, most likely in the form of CDM substructure or a complex lens environment. Determining in practice that a given lens is anomalous requires some care. Equations (11) and (2) show that $R_{\text {fold }}$ and $R_{\text {cusp }}$ increase with the distance between the images, so a non-zero value for one of these quantities does not automatically imply a flux ratio anomaly. Making such an identification would require knowledge of $A_{\text {fold }}$ and

\footnotetext{
5 Strictly speaking, optical and radio emission come from different regions of a quasar, so the optical and radio image positions of a lens, and hence the corresponding time delays, need not be identical. This effect is naturally taken into account by our criterion for matching observed time delays with simulated lenses (see 33).
} 
$A_{\text {cusp }}$, which depend on the exact form of the lens potential. Since these quantities are not directly observable, Keeton et al. (2003, 2005) performed Monte Carlo simulations to determine distributions for $R_{\text {fold }}$ and $R_{\text {cusp }}$ at fixed $d_{1}$ using parameter values appropriate for a realistic population of lens galaxies. With this information, it is possible to determine the probability that an observed lens is anomalous and hence contains small-scale structure.

Oguri (2007) employed a method similar to that of Keeton et al. (2003, 2005) to study time delays, although his emphasis was different from ours. While both studies are based on time delay distributions, Oguri's emphasis was on determining the Hubble constant, whereas we focus on small-scale structure in lens galaxies. In fact, we explicitly remove the Hubble constant from our analysis by working with scaled time delays and time delay ratios. Another difference is that we concentrate our attention mainly on cusp and fold lenses, which are most relevant to the questions we seek to answer.

This paper is organized as follows. In $₫ 2$ we consider how the differential time delay depends on the parameters of the lens potential, and on the shape of the associated (astroid) caustic. The results we present there motivate our Monte Carlo simulations, which we describe in 43. We apply our formalism to the 25 known four-image lenses in \$4 Finally, we present our conclusions in $\$ 5$.

\section{DEPENDENCE OF TIME DELAY ON LENS POTENTIAL} AND POSITION ALONG CAUSTIC

In Congdon et al. (2008) we showed that the time delay between a close pair of images in a fold lens scales with the cube of the image separation, $d_{1}$, i.e., $\Delta \tau / \tau_{0} \approx$ $|h| d_{1}^{3} / 2$ (see eq. [25] of Congdon et al. 2008, and pp. 190191 of Schneider et al. 1992), where $\tau_{0}$ is a cosmologydependent scale factor (see eq. 6] below). The coefficient $h$ comes from a Taylor expansion of the lens potential, $\psi$, and can be written as a particular third derivative: $h \equiv \psi_{222} / 6$, where the subscript "2" indicates differentiation with respect to the coordinate in the image plane that corresponds to the direction perpendicular to the caustic in the source plane, and is evaluated at the point on the critical curve that serves as the coordinate origin (see $\S 3.2$ of Congdon et al. 2008). In this section we study how the time delay for a fold pair depends on the lens potential and the distance between the fold point and the nearest cusp point. This is equivalent to studying the variation of $h$ along the caustic, since, for fixed $d_{1}$, the time delay is given solely in terms of this coefficient. Because $h<0$, we find it more convenient to work with its absolute value.

Most lens galaxies are of early type and have density profiles close to isothermal, i.e., the three dimensional density scales with radius as $\rho \propto r^{-2}$ (e.g., Treu \& Koopmans 2004; Rusin \& Kochanek 2005; Treu et al. 2006), so we compute $|h|$ for a singular isothermal ellipsoid (SIE) lens. To determine an appropriate value for the ellipticity parameter $e \equiv$ $1-q$, where $q$ is the minor-to-major axis ratio, we turn to the observed galaxy samples of Bender et al. (1989), Jørgensen et al. (1995), and Saglia et al. (1993). These samples have mean ellipticities and dispersions of $\left(\bar{e}, \sigma_{e}\right)=(0.28,0.15),(0.31,0.18)$, and $(0.30,0.16)$ respectively. Note that these values measure the distribution
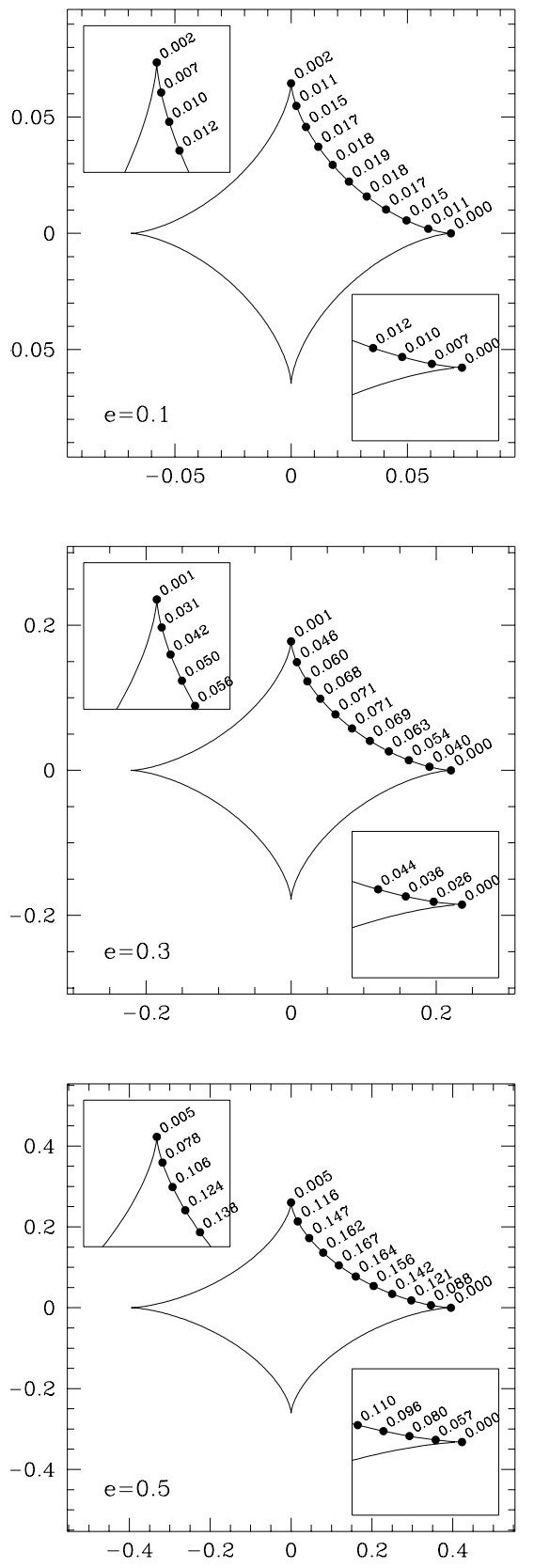

Fig. 1.- Values of the Taylor series coefficient $|h|$ at various points on the caustic for a singular isothermal ellipsoid lens with different values of the ellipticity. Insets show close-up views of the upper and right-hand cusp points. The coefficient $h$ should vanish at a cusp point (see Congdon et al. 2008), but for computational reasons we do not necessarily have points that lie precisely at the cusps. The axes are in units of the Einstein angle.

of light rather than mass, so it is possible that the dark matter halo in which the galaxy presumably resides is rounder or flatter than the observed isophotes.

Figure 1 shows $|h|$ at various points on the caustic, for ellipticities of $0.1,0.3$, and 0.5 . We see that $|h|$ remains roughly constant along the caustic for points away from the cusps. This suggests that lenses whose fold pairs have comparable separations will have similar time delays as well, at least for galaxies with similar ellipticities. 
Testing this prediction will require large samples of fold lenses, for which both the differential time delay between the fold pair and the ellipticity of the lens galaxy are known. For points near a cusp, $|h|$ decreases rapidly and vanishes right at the cusp point (as it must; see $\S 2$ of Congdon et al. 2008). Finally, we see that $|h|$ depends on the size of the caustic. It is not yet clear whether this merely reflects a simple correlation between $|h|$ and $e$ or is indicative of a more subtle relationship between caustic size and the time delay for a fold pair.

In addition to ellipticity, Bender et al. (1989) and Saglia et al. (1993) find that many early-type galaxies have small departures from elliptical symmetry. "Disky" or "boxy" isophotes can be represented by an $m=4$ multipole mode, with coefficient $a_{4}>0$ for disky isophotes and $a_{4}<0$ for boxy isophotes. In terms of the (twodimensional) lens potential, the $m=4$ mode for an isothermal galaxy can be written as (e.g., Keeton 2001)

$$
\psi(r, \theta)=-A_{4} r \cos 4 \theta .
$$

The coefficient $a_{4}$ is defined in terms of surface brightness rather than potential, so we must convert from $A_{4}$ to $a_{4}$ :

$$
a_{4}=15 A_{4} \sqrt{1-\epsilon},
$$

where $\epsilon \equiv\left(1-q^{2}\right) /\left(1+q^{2}\right)$. For typical values of $a_{4}=$ $-0.01,0,0.01$, and 0.02 (e.g., Bender et al. 1989) and ellipticity $e=0.3$, we find maximum $|h|$-values of 0.07 , $0.07,0.08$, and 0.09 , respectively. We therefore conclude that $m=4$ multipole terms have fairly small effects on the time delay for a fold pair, regardless of whether the isophote is disky or boxy. We nevertheless include $m=4$ modes in our simulations (\$3) given that this is both simple and observationally motivated.

Since many lens galaxies lie within groups or clusters (e.g., Momcheva et al. 2006; Auger et al. 2007), a nonzero tidal shear is common. Using numerical simulations and semianalytic models of galaxy formation, Holder \& Schechter (2003) find that shear can be described by a lognormal distribution with median $\gamma=0.05$ and dispersion $\sigma_{\gamma}=0.2$ dex. ${ }^{6}$ For such shear amplitudes, the caustic structure is qualitatively similar to what is seen in Figure 1 so we do not show it explicitly. We return to shear when we consider realistic lens populations in the following section.

\section{CONSTRUCTING TIME DELAY DISTRIBUTIONS FOR A REALISTIC LENS POPULATION}

For any given lens galaxy, we could estimate $h$ (or more generally the smooth model time delays) directly, by fitting a lens model and inferring the lens potential. However, we would like to avoid any dependence on models and modeling as much as possible. We therefore elect to examine the full probability distributions for time delays given a realistic population of lens galaxies, using Monte Carlo methods to construct the time delay distributions.

We follow the approach of Keeton et al. (2003, 2005) and create lens galaxies with model parameters drawn from the galaxy samples of Bender et al. (1989), Jørgensen et al. (1995), and Saglia et al. (1993). Although the mean ellipticities and dispersions are simi-

\footnotetext{
${ }^{6}$ Following Keeton et al. (2005), we do not quote the final shear distribution of Holder \& Schechter (2003), but rather the raw distribution before effects such as magnification bias are applied.
}

lar for the three samples, the underlying galaxy populations are not the same. Jørgensen et al. (1995) and Saglia et al. (1993) include galaxies within clusters, while Bender et al. (1989) use bright, nearby galaxies to construct their sample. In addition, Bender et al. (1989) and Saglia et al. (1993) report $a_{4}$ measurements, while Jørgensen et al. (1995) do not. In order to determine the extent to which our conclusions depend on the input data, we carry out the numerical method described below separately for each of the three galaxy samples. To model the environment of a lens galaxy, we add tidal shear based on the simulations of Holder \& Schechter (2003) that we discussed at the end of the previous section. For the sample of Jørgensen et al. (1995) we pick 2000 ellipticities from their observed distribution, and assign a random shear to each. For the samples of Bender et al. (1989) and Saglia et al. (1993), we use the actual $\left(e, a_{4}\right)$ pairs for the observed galaxies ( 87 in the Bender et al. 1989 sample, and 54 in the Saglia et al. 1993 sample) in order to retain any correlation between the parameters; and for each galaxy we use 100 different realizations of the shear.

For each model lens potential, we use an updated version of the GRAVLENS software ${ }^{7}$ (Keeton 2001) to solve the lens equation numerically and obtain the image positions and time delays for a large set of random source positions that are uniformly distributed in the four-image region. Using a uniform distribution has several consequences. First, it means that each model lens potential is automatically weighted by its four-image cross section, which seems like the proper statistical approach. ${ }^{8}$ Second, it means that we neglect magnification bias. While lensing magnification bias is quite important when comparing statistical samples of four-image and two-image lenses, it is less dramatic within a sample of four-image lenses, and still less so within the subset of four-image lenses that have a particular image configuration. Generally speaking, including magnification bias would give more weight to sources that lie closer to the lensing caustic, which tend to produce shorter time delays, so it would shift the time delay distributions we derive to somewhat shorter values. The effect is not strong, however, and we checked that it does not change any of our conclusions about time delay anomalies. All told, our simulations based on the galaxy samples from Bender et al. (1989), Jørgensen et al. (1995), and Saglia et al. (1993) contain 1,267,555, 2,205,515, and 851,261 mock four-image lens systems, respectively.

The time delay of an image at angular position $\boldsymbol{\theta}$ relative to an unlensed light ray from the true source with position $\boldsymbol{\beta}$ is given by

$$
\tau(\boldsymbol{\theta})=\tau_{0}\left[\frac{1}{2}|\boldsymbol{\theta}-\boldsymbol{\beta}|^{2}-\psi(\boldsymbol{\theta})\right],
$$

where the time scale is

$$
\tau_{0}=\frac{1+z_{L}}{c} \frac{D_{L} D_{S}}{D_{L S}} .
$$

Here $D_{L}, D_{S}$, and $D_{L S}$ are the angular-diameter dis-

\footnotetext{
7 See http://redfive.rutgers.edu/ keeton/gravlens

8 The mass of the lens galaxy factors out when we work with scaled time delays and time delay ratios, but the angular structure
} of the lens potential remains important. 
tances from the observer to lens, observer to source, and lens to source, respectively. For our mock lenses we focus on the dimensionless, scaled time delay

$$
\hat{\tau} \equiv \frac{\tau}{\tau_{0} \theta_{E}^{2}},
$$

where $\theta_{E}$ is the Einstein angle. ${ }^{9}$ The advantage of working with the scaled time delay is that our analysis of the mock lenses is independent of cosmology, and of the lens and source redshifts. It does mean that we need to convert all observed time delays from physical to dimensionless units before we can compare them to our mock lens catalog (see $\$ 4$ ). For convenience, we quote distances (e.g., $d_{1}$ and $d_{2}$ below) in units of the Einstein angle as well.

Our analytic time delay relation (eq. 25 of Congdon et al. 2008) specifically applies to fold image pairs, so they are the most obvious targets for anomaly searches. However, we do not actually use the analytic relation to make predictions for our mock lenses, so it is reasonable to consider non-fold image pairs, as well as cusp and cross lenses, in our comparisons between observed lenses and Monte Carlo simulations. There are six distinct image pairs in a four-image lens. We are interested in the subset of pairs that contain one image of positive parity (which lies at a minimum of the time delay surface) and one of negative parity (which lies at a saddlepoint). In this way, we consider only adjacent images. The effect is to make our analysis local ${ }^{10}$ rather than global, which is appropriate for studying small-scale structure. There are four such mixed-parity image pairs in each four-image lens.

We characterize each image pair by the separation between the two images, $d_{1}$. We also use the distance to the next-nearest image, $d_{2},{ }^{11}$ because it helps specify the lens morphology: a fold lens has $d_{1} \ll d_{2} \sim 1$; a cusp lens has $d_{1} \sim d_{2} \ll 1$; a cross lens has $d_{1} \sim d_{2} \sim 1$. Another reason to use $d_{2}$ is that it allows us to take ratios of time delays (see below). While $d_{1}$ and $d_{2}$ technically define an image triplet, we will use the term "pair" to refer to the two images defined by $d_{1}$, since this is the image pair of primary interest.

We seek to understand the full range of time delays that can be produced by smooth lens models that are consistent with the positions of an observed image pair. We consider a mock lens image pair to "match" an observed pair if two conditions are satisfied. First, we require that the $d_{1}$ and $d_{2}$ values of the mock and observed pairs agree within a tolerance of \pm 0.05 . This criterion is conservative in the sense that we are using minimal information from quantities that are observable and lo-

9 The Einstein angles we use come from lens modeling, either by ourselves or others (see Table 1). Although there is no unique definition for the Einstein angle of an elliptical lens, different definitions result in values consistent to within a factor of order unity. In practice, such concerns are rendered moot by the $5 \%$ astrometric uncertainties we allow for when comparing observed lenses with our mock catalog (see text for details).

10 It would perhaps be better to say that our analysis is quasilocal. It is indeed local for close pairs of images (as in fold pairs and cusp triplets), but it is not strictly local for image pairs whose image separation $d_{1}$ approaches order unity.

11 To obtain $d_{2}$ we consider the distance between each image of a given pair and its next-nearest image of opposite parity. We define $d_{2}$ to be the smaller of these two distances. cal; using additional information would only narrow the range of smooth models that are considered to be consistent with an observed image pair. The distance-based matching criterion does have one limitation, which is illustrated in Figure 2 it may allow not only mock pairs with the same parities as the observed images, but also pairs with the opposite parities. We could avoid this problem by adding information about the fourth image. We do not want to do that, however, because the fourth image is always "far" from the pair in question (i.e., 1-2 Einstein angles away); including it would make our analysis more sensitive to global properties of the lens potential and countermand our goal of using a local analysis to search for substructure. Instead, we add the image parities to the matching criterion. ${ }^{12}$ Specifically, we insist that the parities of the three images defined by $d_{1}$ and $d_{2}$ are the same for an observed lens and its simulated counterpart. This is straightfoward to do since the image parities are known for the mock lenses, and they can be determined unambiguously for observed lenses (by measuring time delays or just analyzing the image configuration; see, e.g., Saha \& Williams 2003). Since the two images in each pair we consider have opposite parities (by construction), the parity cut represents only one additional bit of information.

For each image pair we compute the differential scaled time delay between the two images, which we call $\Delta t_{1}$. For the second differential time delay we use the image pair characterized by the distance $d_{2}$, and we call this $\Delta t_{2}$. We then adopt the following sign convention. By construction, any image pair we consider will consist of a minimum image (positive parity, labeled $M_{1}$ ) and a saddle image (negative parity, labeled $S_{1}$ ). If the nextnearest image is a minimum (labeled $M_{2}$ ), we have a triplet consisting of a saddle flanked by two minima. We define the two differential time delays to be

$$
\Delta t_{1}=\hat{\tau}\left(M_{1}\right)-\hat{\tau}\left(S_{1}\right) \text { and } \Delta t_{2}=\hat{\tau}\left(M_{2}\right)-\hat{\tau}\left(S_{1}\right) .
$$

Since saddles have larger time delays than minima, both $\Delta t_{1}$ and $\Delta t_{2}$ are negative. In the case that the nextnearest image is a saddle (labeled $S_{2}$ ), we have a triplet consisting of a minimum flanked by two saddles. We then define the differential time delays to be

$$
\Delta t_{1}=\hat{\tau}\left(S_{1}\right)-\hat{\tau}\left(M_{1}\right) \text { and } \Delta t_{2}=\hat{\tau}\left(S_{2}\right)-\hat{\tau}\left(M_{1}\right) .
$$

Now both $\Delta t_{1}$ and $\Delta t_{2}$ are positive.

It is also useful to compute the ratio of the differential time delays for two image pairs. Time delay ratios are attractive because they do not depend on cosmology ( $\tau_{0}$ factors out), and they are largely immune to the radial-profile degeneracy (e.g., Kochanek 2002; Keeton \& Moustakas 2009). Our sign convention ensures that $\Delta t_{1} / \Delta t_{2}$ is always positive since $\Delta t_{1}$ and $\Delta t_{2}$ have the same sign.

To reprise, we define the two time delays relative to the "middle" of the three images we use to determine $d_{1}$ and $d_{2}$. This means the scaled time delays can be either positive or negative, but the time delay ratio is always positive. Note that regardless of the sign, when labeling image pairs we always list the minimum first.

\footnotetext{
12 The parity cut was not used by Keeton et al. (2003, 2005), but we introduce it here in part because Keeton \& Moustakas (2009) note the importance of parity in the context of time delays.
} 

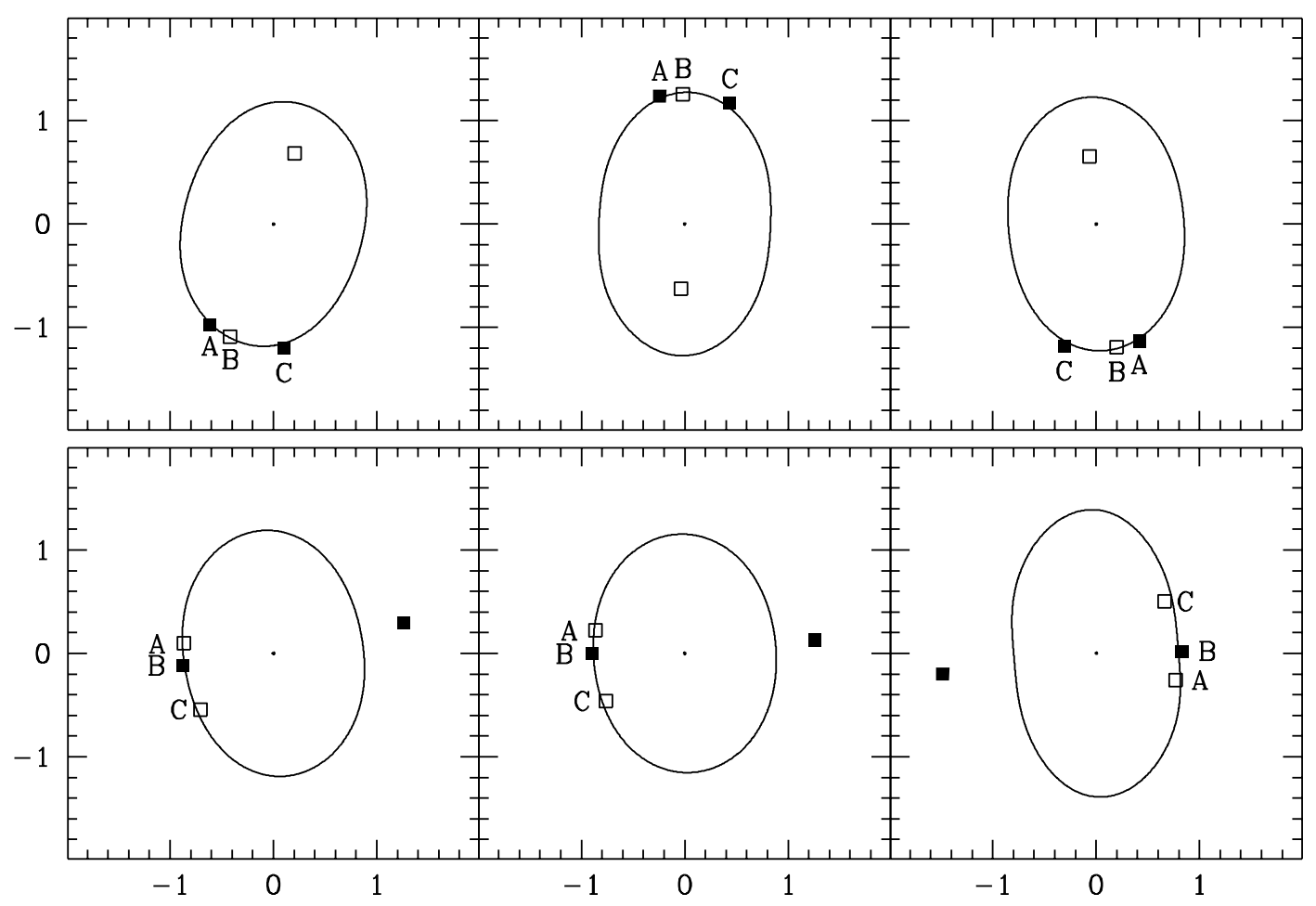

FIG. 2.- Examples of mock lenses that potentially match the cusp lens B2045+265. Specifically, the distances $d_{1}$ (between images A and $\mathrm{B}$ ) and $d_{2}$ (between images $\mathrm{B}$ and C) match the corresponding distances in 2045 within a tolerance of \pm 0.05 . Filled squares denote minima, while open squares denote saddles. The lensing critical curves are shown, and the axes are labeled in units of the Einstein angle, $\theta_{E}$. The major axis of the lens galaxy is oriented vertically, so the mock lenses in the top row are long-axis cusps, while the mock lenses in the bottom row are short-axis cusps. When the parity cut is applied, only the top panels match 2045.

From the set of mock lenses that match an observed image pair, we construct histograms of the scaled time delay and the time delay ratio. These represent the range of values that can be produced by realistic smooth mass distributions. We can then use these predicted distributions to assess whether observed time delays are or are not consistent with lensing by a smooth mass distribution.

\section{RESULTS FOR OBSERVED LENSES}

The number of observed four-image lenses has been steadily increasing in recent years. We focus on the 25 lens systems for which the lensed images are point-like (see Table 11). This restriction prevents us from using the system Q0047-2808 (Warren et al. 1996, 1999), as well as lenses from the SLACS survey ${ }^{13}$ (Bolton et al. 2006, 2008). However, this condition is necessary to ensure that the lensed source is compact, so that the variability timescale is short enough to make time delay measurements practical.

As noted above (see eq. 7), we compute scaled time delays for our mock lenses, so we must scale each observed time delay by $\tau_{0} \theta_{E}^{2}$ in order to compare it with the mock lens sample. The Einstein angles and lens and source redshifts are listed in Table 1. We compute the angular diameter distances in $\tau_{0}$ assuming cosmological parameters $\Omega_{M}=0.3, \Omega_{\Lambda}=0.7$, and $H_{0}=70 \mathrm{~km} \mathrm{~s}^{-1}$ $\mathrm{Mpc}^{-1}$. The benefit of working with scaled time delays in our mock lens analysis is that we can present results

\footnotetext{
13 See http://www.slacs.org/
}

for all observed lenses, regardless of whether any of their time delays are currently known. As more time delays are measured, it will be a simple matter to compare them with our compilation of predicted scaled time delays. In addition, it is possible to consider different cosmological models simply by recomputing the scale factor $\tau_{0}$ and retranslating between observed and scaled time delays.

\subsection{Time Delay Histograms}

To get a sense of the range of time delays that can be produced by smooth lens models, we show histograms of the scaled time delay (Figs. (3+6) and time delay ratio (Figs. 7][10) for each image pair in the 25 known fourimage lenses. There are many lenses whose time delays have not yet been measured, but the histograms for those cases are still pedagogically useful and provide a way to predict what the time delay should be if the lens in question is not anomalous. In this subsection we discuss the general features of the histograms. In the following subsections we analyze the lenses with known time delays and make predictions for the remainder.

We divide the observed lenses into three groups: folds (Figs. 31 4, 7, and 8), cusps (Figs. 5) and 9), and crosses (Figs. 6 and 10). We assign one of these canonical lens morphologies to each observed four-image lens according to the classification scheme of Keeton et al. (2003, 2005). For each lens, we define $d_{1}^{*}$ to be the smallest value of the pairwise image separations. The lenses in each figure are arranged in rows such that $d_{1}^{*}$ increases from top to bottom. Within each row, the panels are arranged such that $d_{1}$ increases from left to right. As a check for 
TABLE 1

DATA FOR FOUR-IMAGE LENSES

\begin{tabular}{lrrrr}
\hline \hline Lens Name & $z_{S}$ & $z_{L}$ & $\theta_{E}(\operatorname{arcsec})$ & $\tau_{0}\left(\right.$ days $\left.\operatorname{arcsec}^{-2}\right)$ \\
\hline B0128+437 & 3.12 & $?$ & 0.20 & $?$ \\
HE 0230-2130 & 2.16 & 0.52 & 0.82 & 85.7 \\
MG 0414+0534 & 2.64 & 0.96 & 1.08 & 193.2 \\
B0712+472 & 1.34 & 0.41 & 0.68 & 72.9 \\
HS 0810+2554 & 1.50 & $?$ & 0.51 & $?$ \\
SDSS 0924+0219 & 1.52 & 0.39 & 0.87 & 64.8 \\
SDSS J1004+4112 & 1.73 & 0.68 & 6.91 & 140.7 \\
PG 1115+080 & 1.74 & 0.31 & 1.03 & 46.5 \\
SDSS J1330+1810 & 1.39 & 0.37 & 0.97 & 62.8 \\
B1555+375 & $?$ & $?$ & 0.24 & $?$ \\
B1608+656 & 1.39 & 0.63 & 0.77 & 143.7 \\
B1933+503 & 2.63 & 0.76 & 0.49 & 135.8 \\
WFI 2026-4536 & 2.23 & $?$ & 0.65 & $?$ \\
WFI 2033-4723 & 1.66 & 0.66 & 1.06 & 137.6 \\
\hline RX J0911+0551 & 2.80 & 0.77 & 0.95 & 65.0 \\
RX J1131-1231 & 0.66 & 0.30 & 1.81 & 46.1 \\
SDSS J1251+2935 & 0.80 & 0.41 & 0.88 & 342.1 \\
B1422+231 & 3.62 & 0.34 & 0.76 & $?$ \\
B2045+265 & 1.28 & 0.87 & 1.13 & $?$ \\
HE 0435-1223 & 1.69 & 0.46 & 1.18 & $?$ \\
HST 12531-2914 & $?$ & 0.69 & 0.55 & 135.7 \\
HST 14113+5211 & 2.81 & 0.46 & 0.83 & 4.9 \\
H1413+117 & 2.55 & $?$ & 0.56 & \\
HST 14176+5226 & 3.40 & 0.81 & 1.33 & \\
Q2237+030 & 1.69 & 0.04 & 0.85 & \\
& & & &
\end{tabular}

Note. - Data for the known four-image lenses with pointlike images. All data are given by Oguri (2007) and the CASTLES website (http://www.cfa.harvard.edu/castles/), except for SDSS J1330+1810 (Oguri et al.2008) and SDSS J1251+2935 (Kavo et al. 2007). Question marks indicate quantities for which no measured value is available. Einstein angles $\theta_{E}$ are computed from lens models, either our own or others' (CASTLES, Keeton et al. 2003, 2005; Kavo et al. 2007; Oguri et al. 2008). The timescale $\tau_{0}$ depends on $z_{L}$ and $z_{S}$. We assume cosmological parameters $\Omega_{M}=0.3, \Omega_{\Lambda}=0.7$, and $H_{0}=70 \mathrm{~km} \mathrm{~s}^{-1} \mathrm{Mpc}^{-1}$. The table is divided into three sections: fold lenses (top), cusp lenses (middle), and cross lenses (bottom).

systematic effects, we plot histograms of the time delays and time delay ratios produced by Monte Carlo simulations using galaxy samples from Bender et al. (1989), Jørgensen et al. (1995), and Saglia et al. (1993) (see \$3).

We first consider histograms of the scaled time delays, which are shown in Figures [3,6. A negative time delay indicates that the middle image in the triplet defined by $d_{1}$ and $d_{2}$ has negative parity (eq. 8]), while a positive time delay indicates a middle image with positive parity (eq. 9). The histograms are not highly sensitive to the input galaxy sample, especially in the tails (which are most important for identifying anomalies). The histograms are somewhat asymmetric with a longer tail on the side away from zero, which presumably reflects the fact that the histograms are bounded by zero by construction. Many of the histograms have a large width relative to the mean/median, which is not too surprising because we have been quite generous in matching mock lenses to observed lenses on the basis of minimal information (just $d_{1}, d_{2}$, and parity). This is consistent with our goal of being conservative in identifying time delay anomalies.

Next, we consider histograms of the time delay ratios, which are shown in Figures 7 10. These histograms are skewed to the right, presumably because the time delay ratios are positive and hence the histograms are bounded by zero on the left but unbounded on the right. The overall structure of the time delay ratio histograms does not vary much from one lens to another, or between image pairs of a given lens. This suggests that conclusions drawn from time delay ratios are not terribly sensitive to the lens morphology (fold, cusp, or cross), which may prove quite useful.

\subsection{Identifying Time Delay Anomalies in Observed Lenses}

Eight of the 25 known four-image lenses have at least one image pair with an observed time delay (see Tables 2 and 3). We can use our simulations to determine whether these lenses are consistent with lensing by an ellipsoidal mass distribution with tidal shear. Specifically, we compare the observed value of the time delay and (if available $)^{14}$ the time delay ratio with our predicted distributions. If the observed value lies outside of the predicted range, we classify the time delay as anomalous and interpret it as strong evidence that the lens galaxy cannot be described as a simple, relatively smooth mass distribution with shear.

\footnotetext{
14 Recall that to construct the time delay ratio we must know not only the time delay for the image pair, but also the time delay to the next nearest image.
} 
TABLE 2

P-VAlues FOR SCALED TIME DELAYS

\begin{tabular}{|c|c|c|c|c|c|c|}
\hline $\begin{array}{l}\text { Lens } \\
\text { Name }\end{array}$ & $\begin{array}{l}\text { Image } \\
\text { Pair }\end{array}$ & Rank & $\begin{array}{r}\text { Obs. } \Delta t_{1} \\
(\text { days })\end{array}$ & $\begin{array}{c}\text { Error } \\
\text { Interval }\end{array}$ & $\begin{array}{l}\text { P-value } \\
\text { for } \Delta t_{1}\end{array}$ & $\begin{array}{l}\text { P-values for } \\
\text { Err. Interval }\end{array}$ \\
\hline 1004 & $\mathrm{BA}$ & 1 & 40.6 & $(35.2,46.0)$ & 0.114 & $(0.0630,0.170)$ \\
\hline 1004 & $\mathrm{CA}$ & 3 & -822. & $(-828 .,-815)$. & 0.917 & $(0.914,0.919)^{\prime}$ \\
\hline 1115 & $\mathrm{~A} 1 \mathrm{~A} 2$ & 1 & 0.149 & $(0.131,0.167)$ & 0.109 & $(0.0670,0.154)$ \\
\hline 1115 & A1B & 2 & 11.7 & $(8.10,15.3)$ & 0.292 & $(0.121,0.487)$ \\
\hline 1115 & $\mathrm{CB}$ & 3 & -25.0 & $(-29.8,-20.2)$ & 0.983 & $(0.922,0.996)$ \\
\hline 1115 & CA2 & 4 & -13.3 & $(-16.3,-10.3)$ & 0.991 & $(0.971,0.998)$ \\
\hline 1608 & $\mathrm{AC}$ & 1 & -4.50 & $(-9.00,0)$ & 1.00 & $(0.995,1.00)$ \\
\hline 1608 & $\mathrm{BC}$ & 2 & -36.0 & $(-40.5,-31.5)$ & 0.921 & $(0.849,0.965)$ \\
\hline 1608 & $\mathrm{AD}$ & 3 & 45.5 & $(41.0,50.0)$ & 0 & $(0,0)$ \\
\hline 1608 & BD & 4 & 77.0 & $(71.0,83.0)$ & - & $(-,-)$ \\
\hline 2033 & $\mathrm{~A} 1 \mathrm{C}$ & 2 & 27.1 & $(14.2,40.0)$ & 0.469 & $(0.0882,0.791)$ \\
\hline 2033 & BA2 & 3 & -35.5 & $(-39.7,-31.3)$ & 0.903 & $(0.843,0.945)^{\prime}$ \\
\hline 2033 & $\mathrm{BC}$ & 4 & -62.6 & $(-74.9,-50.3)$ & 0.998 & $(0.995,1.00)$ \\
\hline 0911 & BA & 1 & 6.00 & $(-24.0,36.0)$ & 1.00 & $(0,1.00)$ \\
\hline 0911 & $\mathrm{BC}$ & 2 & 5.00 & $(-48.7,58.7)$ & 1.00 & $(0,1.00)$ \\
\hline 0911 & $\mathrm{DC}$ & 3 & -154 & $(-202 .,-106)$. & 1.00 & $(1.00,1.00)$ \\
\hline 0911 & DA & 4 & -143. & $(-161 .,-125)$. & 1.00 & $(1.00,1.00)$ \\
\hline 1131 & $\mathrm{BA}$ & 1 & -12.0 & $(-16.5,-7.50)$ & 0 & $(0,0)$ \\
\hline 1131 & $\mathrm{CA}$ & 2 & -9.60 & $(-15.6,-3.60)$ & 0 & $(0,0.00601)$ \\
\hline 1131 & $\mathrm{BD}$ & 3 & 99.0 & $(75.0,123)$. & 0.455 & $(0.266,0.632)$ \\
\hline 1131 & CD & 4 & 96.6 & $(72.6,121)$. & 0.376 & $(0.206,0.548)$ \\
\hline 1422 & $\mathrm{AB}$ & 1 & -1.50 & $(-5.70,2.70)$ & 0 & $(0,1.00)$ \\
\hline 1422 & $\mathrm{CB}$ & 2 & -8.20 & $(-14.2,-2.20)$ & 0 & $(0,0.000361)$ \\
\hline 0435 & $\mathrm{CB}$ & 1 & -5.90 & $(-8.30,-3.50)$ & 0.931 & $(0.773,0.991)$ \\
\hline 0435 & $\mathrm{AB}$ & 2 & -8.00 & $(-10.4,-5.60)$ & 0.862 & $(0.676,0.963)$ \\
\hline 0435 & CD & 3 & 12.3 & $(9.90,14.7)$ & 0.0489 & $(0.0205,0.0907)$ \\
\hline 0435 & $\mathrm{AD}$ & 4 & 14.4 & $(11.7,17.1)$ & 0.0668 & $(0.0307,0.119)$ \\
\hline
\end{tabular}

Note. - Column 1 gives abridged lens names (see Table 1 for the corresponding full names). Image pairs contain one minimum and one saddle. The labels in column 2 list the minimum image first. We rank image pairs according to their separation in column 3, with smaller numbers corresponding to smaller separations. Columns 4 and 5 list the observed time delays, along with their $3 \sigma$ error bars. In cases where the measurement uncertainties are asymmetric about the observed value, we create symmetric error bars with uncertainty $\sigma$, where $\sigma$ refers to the larger of the two measurement uncertainties. Note that the parity cut described in $\$ 3$ specifies the sign of the time delay in a given image pair, so time delays with the opposite sign are unphysical even if such values are formally allowed by the measurement uncertainties. The observational time delay data used here can be found in Table 1 of Oguri (2007), except for the lenses 1004 (Fohlmeister et al. 2008) and 2033 (Vuissoz et al. 2008). Columns 6 and 7 give $\mathrm{P}$-values for the time delays shown in columns 4 and 5, using the galaxy sample of Bender et al. (1989).

This statement is quantified by the statistical P-value, which gives the fraction of matching mock lenses whose time delays (or ratios) are smaller than the observed value; either $P>0.995$ or $P<0.005$, for example, would indicate that a time delay is anomalous at more than $99 \%$ confidence. We consider $P>0.995$ or $P<0.005$ to indicate a strong anomaly, and $0.975<P<0.995$ or $0.005<P<0.025$ to indicate a marginal anomaly. Given the relatively small number of known four-image lenses, we should not read too deeply into marginal anomalies since a few outliers are only to be expected. The extreme $\mathrm{P}$-values required of strong anomalies, however, would be difficult to interpret as statistical flukes, so conclusions based on such systems should be robust. The choice of input galaxy sample for the Monte Carlo simulations has no significant effect on our conclusions; so for simplicity we report $\mathrm{P}$-values computed using only the sample of Bender et al. (1989), which is the larger of the two samples that include $a_{4}$ measurements.

To assess whether measurement errors in observed time delays affect our results, we compute P-values for the endpoints of the observed $3 \sigma$ error interval. This is a simple task for time delays, but for time delay ratios we first need to propagate errors from the time delays into the ratios. For time delay errors that are symmetric, we assume they are Gaussian and propagate them using the standard formula for a quotient. If the errorbars are asymmetric, we conservatively take the larger errorbar as the Gaussian standard deviation, $\sigma$. While this approach is not strictly correct, it is the best we can do without knowing the full error distribution, and it is conservative in the sense that it should overestimate the uncertainties in the time delay ratio. It turns out that our identifications of anomalies are not affected by the observational errors in most cases (see Tables 2 and 3 , and the following discussions of individual lenses). This does not mean, however, that there is no need to measure time delays more precisely. Rather, it reflects the fact that our predicted time delay distributions are quite broad because we have deliberately chosen to be generous 
TABLE 3

P-VALUES FOR TIME DELAY RATIOS

\begin{tabular}{lcrrcrc}
\hline \hline $\begin{array}{l}\text { Lens } \\
\text { Name }\end{array}$ & $\begin{array}{c}\text { Image } \\
\text { Pair }\end{array}$ & Rank & $\begin{array}{r}\text { Observed } \\
\Delta t_{1} / \Delta t_{2}\end{array}$ & $\begin{array}{c}\text { Error } \\
\text { Interval }\end{array}$ & $\begin{array}{r}\text { P-value } \\
\text { for } \Delta t_{1} / \Delta t_{2}\end{array}$ & $\begin{array}{c}\text { P-values for } \\
\text { Err. Interval }\end{array}$ \\
\hline 1004 & CA & 3 & 20.2 & $(17.5,22.9)$ & 0.0646 & $(0.0139,0.161)$ \\
1115 & A1A2 & 1 & 0.0127 & $(0.00853,0.0169)$ & 0.178 & $(0.0253,0.445)$ \\
1115 & A1B & 2 & 78.5 & $(52.6,104)$. & 0.751 & $(0.364,0.916)$ \\
1115 & CB & 3 & 2.14 & $(1.36,2.91)$ & 0.755 & $(0.0543,0.962)$ \\
1115 & CA2 & 4 & 89.3 & $(66.4,112)$. & 0.344 & $(0.130,0.527)$ \\
1608 & AC & 1 & 0.125 & $(-0.000973,0.251)$ & 0 & $(0,0.696)$ \\
1608 & BC & 2 & 8.00 & $(-0.0623,16.1)$ & 0.706 & $(0,0.987)$ \\
1608 & AD & 3 & 10.1 & $(-0.0493,20.3)$ & 0.177 & $(0,0.967)$ \\
1608 & BD & 4 & 2.14 & $(1.82,2.45)$ & & $(-1.3)$ \\
2033 & BC & 4 & 2.31 & $(1.12,3.50)$ & 0.193 & $(0,0.746)$ \\
0911 & BA & 1 & 1.20 & $(-13.0,15.4)$ & 0.992 & $(0,1.00)$ \\
0911 & BC & 2 & 0.833 & $(-9.04,10.7)$ & 0.00753 & $(0,1.00)$ \\
0911 & DC & 3 & 30.8 & $(-300 ., 362)$. & 0 & $(0,0.987)$ \\
0911 & DA & 4 & 23.8 & $(-95.4,143)$. & 0 & $(0,0.0200)$ \\
1131 & BA & 1 & 1.25 & $(0.339,2.16)$ & 0.976 & $(0,1.00)$ \\
1131 & CA & 2 & 0.800 & $(0.217,1.38)$ & 0.0240 & $(0,0.785)$ \\
1131 & BD & 3 & 8.25 & $(4.57,11.9)$ & 0.000404 & $(0,0.00623)$ \\
1131 & CD & 4 & 10.1 & $(3.29,16.8)$ & 0.00388 & $(0,0.0589)$ \\
1422 & AB & 1 & 0.183 & $(-0.346,0.712)$ & 0.0350 & $(0,1.00)$ \\
1422 & CB & 2 & 5.47 & $(-10.4,21.3)$ & 0.965 & $(0,1.00)$ \\
0435 & CB & 1 & 0.738 & $(0.365,1.11)$ & 0.157 & $(0.000215,0.861)$ \\
0435 & AB & 2 & 1.36 & $(0.671,2.04)$ & 0.843 & $(0.00951,0.996)$ \\
0435 & CD & 3 & 2.08 & $(1.14,3.03)$ & 0.378 & $(0.0151,0.826)$ \\
0435 & AD & 4 & 1.80 & $(1.16,2.44)$ & 0.275 & $(0.0216,0.635)$ \\
\hline
\end{tabular}

Note. - Columns 1-3 have the same meaning as in Table 2 Columns 4 and 5 list time delay ratios and their corresponding error intervals. Errors on time delay ratios are computed by propagating errors from observed time delays (see 4.2 for details). Note that negative values in column 5 are unphysical and have only formal meaning (see Table 2). Columns 6 and 7 give $\mathrm{P}$-values for the time delay ratios shown in columns 4 and 5, using the galaxy sample of Bender et al. (1989).

in matching observed and mock lenses. When it comes to analyzing time delay anomalies to extract physical information (about the substructure mass function, for example), it will be necessary to do detailed modeling for which precise image positions and time delays will be vital (see Keeton \& Moustakas 2009 and Moustakas et al. 2009 for more discussion).

We now discuss all of the lenses with at least one observed time delay in the order in which they appear in Figures 36 .

$P G$ 1115+080. All of the differential time delays in the fold lens 1115 are known (Schechter et al. 1997; Barkana 1997; Chartas et al. 2004). The flux ratio between the close images A1 and A2 is anomalous at optical and X-ray wavelengths (see Pooley et al. 2007, and references therein). The mid-IR flux ratios are not anomalous, however, which suggests that the X-ray/optical anomaly is caused by microlensing (Chiba et al. 2005). Since microlensing does not affect time delays (Keeton \& Moustakas 2009), we might expect the time delays not to be anomalous. Indeed, Figure 3 and Tables 2 and 3 show that the A1A2 time delay is not anomalous. We now turn to the time delays between the "distant" image pairs. The P-values of the pairs CA2 and CB would be anomalous if we considered only the $1 \sigma$ errorbars, but they fall below the threshold when we use $3 \sigma$ errorbars. Since there are no time-delay anomalies in 1115, we conclude that the observed optical flux anomalies are indeed due to microlensing.

SDSS J1004+4112. The fold lens 1004 is produced by a cluster of galaxies and contains five lensed images whose temporal ordering is C-B-A-D-E (Fohlmeister et al. 2008). Images $\mathrm{C}$ and $\mathrm{B}$ are minima, $\mathrm{A}$ and $\mathrm{D}$ are saddles, and $\mathrm{E}$ is a maximum (Inada et al. 2005b). This is the only quad lens whose maximum (doublynegative parity) image has been observed, so we have not included maxima in our analysis. The time delays are known for the image pairs BA and CA, so we can examine the corresponding scaled time delays and also the time delay ratio for the pair $\mathrm{CA}$; but the other time delays and ratios have not yet been determined. The $\mathrm{P}$-values for the known time delays do not indicate anomalies (according to our 95\% confidence criterion). This seems surprising, because the actual lens potential is presumably very different from our assumed model of an isothermal galaxy with shear: clusters are not expected to have isothermal profiles (e.g., Navarro et al. 1997), and the galaxies in the cluster create significant complexity in the potential. We believe the results for 1004 indicate that our method for finding time delay anomalies is conservative.

WFI 2033-4723. For the fold lens 2033, Vuissoz et al. (2008) report the time delays between images B and $\mathrm{C}$ and between $\mathrm{B}$ and the combination of the close images A1 and A2. The time delay between A1 and A2 was too small to be measured; it is expected to be short enough that we can assume A1 and A2 have effectively the same light travel time for the purpose of determining the time delays for the image pairs $\mathrm{A} 1 \mathrm{C}$ and BA2. Among the distant image pairs, the BC pair has the largest separation and also a strong time delay 
anomaly, while the two pairs with smaller distances are not anomalous. The nominal value of the time delay ratio for the $\mathrm{BC}$ pair does not indicate an anomaly, although the uncertainties are large enough that the situation is not conclusive at present.

The time delay in BC may be affected by the group of galaxies of which the main lens is a member (Morgan et al. 2004; Vuissoz et al. 2008). We find two lines of evidence supporting this hypothesis. First, the time delays become more anomalous as the image separation increases. Second, our predicted time delays are longer than the observed time delays; adding environmental effects to the lens model would generally reduce the predicted time delays. A group would contribute a non-negative convergence $\kappa_{\text {env }}$ to the lens potential, which would rescale the predicted time delays by a factor $1-\kappa_{\text {env }}<1$, and it could also create higher-order effects that may be more complicated (Keeton \& Zabludoff 2004). This and alternative hypotheses that the time delays are affected by a change in the radial profile of the lens galaxy or even the global value of the Hubble constant are discussed below.

$B 1608+656$. All of the differential time delays are known for the fold lens 1608 (Fassnacht et al. 2002). Because there are two galaxies inside the Einstein angle, this lens is not necessarily expected to be well described by our models. Indeed, it is extremely hard for single-galaxy models (even with shear) to reproduce the $d_{1}$ and $d_{2}$ values for the image pair BD: we find only one mock image pair that matches the observed values. We are therefore unable to compute $\mathrm{P}$-values for the $\mathrm{BD}$ time delay (cf. Table 21).

Among the other image pairs, the fold pair AC and the distant pair $\mathrm{AD}$ are anomalous in terms of their scaled time delays. All of these anomalies are very strong; in fact, the $\mathrm{AD}$ scaled time delay and its $3 \sigma$ values have $\mathrm{P}$-values that are strictly zero, meaning there are no matching mock lenses whose time delays are more extreme than the observed values. We note that these anomalies do not necessarily reveal CDM substructure, because the presence of two lens galaxies makes the lens potential more complicated than we have allowed for here. Inferences about substructure need to be done in the context of models that treat this complex lens in more detail (e.g., Koopmans et al. 2003; Suyu et al. 2009).

$R X$ J0911+0551. We now turn to the cusp lenses. Hjorth et al. (2002) report the time delays between each of the three cusp images (A, B, and C) and the fourth image (D) for 0911. We find clear evidence of time delay anomalies in the DC and DA image pairs, which are the two pairs with the largest separations. Since the lens galaxy in 0911 is part of a cluster (Kneib et al. 2000), it is possible that these anomalies are due to environmental effects. Pinning down the origin of the anomalies (i.e., the environment of the lens, or substructure) will require that the time delays among the cusp images be precisely measured. ${ }^{15}$ Although the

\footnotetext{
${ }^{15}$ Chartas et al. (2001) and Morgan et al. (2006) have measured time delays among close images in other lens systems, so we are hopeful that it will be possible to do so in 0911.
}

close time delays can be inferred from current data, the $\mathrm{P}$-values they predict span the range $(0,1)$ when $3 \sigma$ measurement uncertainties are included. At this point we conclude that the 0911 time delays are very intriguing and warrant further study.

$R X$ J1131-1231. Morgan et al. (2006) report the time delays among the close images $\mathrm{A}, \mathrm{B}$, and $\mathrm{C}$ for the cusp lens 1131, along with an estimate of the time delay to the distant image $D$. They note that the time delays among the close images are much longer than expected for a smooth mass distribution, and suggest that the time delays indicate the presence of a massive $\left(\sim 5 \times 10^{10} M_{\odot}\right)$ clump near image $\mathrm{A}$. Keeton \& Moustakas (2009) highlight a second peculiarity of the 1131 time delays, namely that the minimum image $\mathrm{B}$ is observed to lead the minimum image $\mathrm{C}$, whereas smooth models predict the reverse. They suggest that a population of clumps could reverse the temporal ordering of the two minimum images in a cusp lens.

We cannot directly address the issue of the temporal ordering of images $\mathrm{B}$ and $\mathrm{C}$, because we do not consider the time delay between images with the same parity. However, we can offer a more model-independent assessment of the time delays for the other image pairs. We find that the time delays for the cusp pairs BA and CA are anomalous: accounting for $3 \sigma$ errorbars, the $\mathrm{P}$-value for BA is strictly zero, while the pair CA falls just short of a strong anomaly (0.006 compared with the threshold of 0.005 ). This strengthens the conclusion by Morgan et al. (2006) that the observed time delays are not at all consistent with lensing by a reasonable smooth mass distribution. Interestingly, we also find that the distant image pair BD has an (almost strongly) anomalous time-delay ratio, even though its scaled time delay is not anomalous. We note that this anomaly is caused by the observed value being smaller than most of the predicted values; this might be understood in terms of the BA time delay, which appears in the denominator of the BD time-delay ratio, being anomalously longer (in absolute value) than expected. Our main conclusion is that the 1131 time delays are highly anomalous, and this general conclusion together with the specific analyses of Morgan et al. (2006) and Keeton \& Moustakas (2009) strongly suggests that this lens contains significant substructure. We also note, however, that we must be careful when interpreting time-delay ratios, since an anomaly may result from the denominator being anomalous rather than the pair of interest.

B1422+231. For 1422, the nominal values of the time delays (Patnaik \& Narasimha 2001) among the three cusp images $\mathrm{A}, \mathrm{B}$, and $\mathrm{C}$ indicate strong anomalies. However, the uncertainties in the claimed time delays are not much smaller than the time delays themselves, so conclusive statements are impossible at this point. (Notice, for example, that the range of $\mathrm{P}$-values for the AB pair spans 0 to 1.00 given the uncertainties.) The system clearly warrants further study, especially since it is well-known to have anomalous flux ratios (Mao \& Schneider 1998; Bradač et al. 2002). 
HE 0435-1223. Finally, we consider the cross lens 0435 . whose time delays were measured by Kochanek et al. (2006a). The cross image configuration features no close pairs of images, so all the time delays could be affected by substructure or large-scale complexity in the lens potential, or both. We do not find anomalies in any of the image pairs, suggesting that the anomalous fluxes at the $\sim 0.2 \mathrm{mag}$ level in images $\mathrm{A}$ and $\mathrm{C}$ are most likely due to microlensing (Kochanek et al. 2006a) rather than substructure (Morgan et al. 2005). Given our goal of being conservative, it is reassuring to find that a cross lens like 0435 has perfectly reasonable time delays.

In the preceding discussion of individual lenses we have interpreted time delay anomalies as evidence for complex structure in the lens potential. Here we briefly consider three alternative interpretations. One possibility is that the Hubble constant, which is needed to compare observed and predicted time delays, differs from our assumed value of $H_{0}=70 \mathrm{~km} \mathrm{~s}^{-1} \mathrm{Mpc}^{-1}$. A second possibility is that a lens has a mass sheet, or external convergence $\kappa_{\text {ext }}$, whose effects we have neglected (Keeton \& Zabludoff 2004). The apparent anomalies in 2033 and 0911 arise because the model predictions are longer than the observed time delays. Since predicted time delays scale as $\Delta t \propto\left(1-\kappa_{\text {ext }}\right) H_{0}^{-1}$, we would need either to have a strong negative mass sheet or to increase $H_{0}$ in order to eliminate the anomalies. Focusing on the $H_{0}$ possibility, in order to reduce the predicted time delays such that the $95 \%$ confidence intervals overlap the observed values in 2033 and 0911, we would require an $H_{0}$ value in excess of $100 \mathrm{~km} \mathrm{~s}^{-1} \mathrm{Mpc}^{-1}$, which does not seem like a viable option. The lens 1131 is a bit different, because the predicted time delays are shorter than the observed values, which would imply a positive mass sheet or a smaller Hubble constant than we have assumed. Regardless of whether the required mass sheet or $H_{0}$ value is reasonable, however, the existing evidence strongly implies that 1131 does contain substructure (Morgan et al. 2006; Keeton \& Moustakas 2009).

A third possibility is that the radial density profiles of the lens galaxies differ from the isothermal profile we have assumed. Changing the radial profile has more complicated effects on the predicted time delays, but Kochanek (2002) argues that to leading order the changes can be approximated with the scaling $\Delta t \propto(1-\langle\kappa\rangle)$, where $\langle\kappa\rangle$ is the mean convergence in the vicinity of the Einstein angle (specifically, in the annulus spanned by the images). Using this scaling, we can estimate the radial profile that would be required if we wanted to reduce the predicted time delays so the $95 \%$ confidence intervals overlap the observed values. (This analysis only yields an estimate, because the scaling is only approximate and does not include the full complexity of ellipsoidal mass distributions; but it is still instructive.) If we write the 3 -d density profile as $\rho \propto r^{-\eta}$, we estimate that we would need $\eta \approx 1.7$ for 2033 , and $\eta \approx 1.5$ for 0911, compared to $\eta=2$ for an isothermal distribution. For comparison, Koopmans et al. (2006) find that lens galaxies have a mean power law index of $\langle\eta\rangle=2.01_{-0.03}^{+0.02}$, with an RMS scatter of 0.12. Again, the simple analysis here needs to be interpreted with some care, but it does suggest that changing the radial profile does not provide a compelling explanation for the apparent time delay anomalies.

\subsection{Predictions for the Remaining Lenses}

To complete our analysis, we present predictions of the scaled time delays (Table 4) and time delay ratios (Table 5) for all mixed-parity image pairs in all 25 known four-image lenses. Specifically, we use the simulations based on the galaxy sample of Bender et al. (1989) to compute the median value, $95 \%$ confidence interval, and 99\% confidence interval for each quantity for each image pair. These results give a sense of what the time delays should be for lenses that are adequately described as ellipsoidal mass distributions with tidal shear. (See Saha et al. 2006 for a complementary approach based on fitting pixellated mass models to individual lenses.) There is currently great interest in lens monitoring (e.g., Courbin 2003; Eigenbrod et al. 2005; Kochanek et al. 2007; (Moustakas et al. 2008, 2009), and we hope our predictions will be useful in planning observational campaigns to measure time delays. Furthermore, as new time delays are measured, it will be a simple matter to compare them with our predictions to determine whether the time delays are anomalous in a way that indicates a complex lens potential (due either to substructure or to the lens environment).

\section{CONCLUSIONS}

We have introduced a new method to use gravitational lens time delays to detect complex structure in the lens potential. The complexity may be associated with CDM substructure, in which case time delays offer the chance to learn more about the substructure population than is possible with lens flux ratios; or it may be associated with the lens environment, such as a group or cluster of galaxies surrounding the lens. The basic approach is to determine the range of time delays that can be produced by reasonable smooth lens models, so that we can identify outliers as being anomalous. To get a sense of how this could work, we first studied the dependence of the time delay between the close pair of images in a fold lens on the position of the source and the form of the lens potential. For a source near a fold point, we have found that the time delay remains approximately constant as the source moves along the caustic. For a lens modeled by an elliptical galaxy with $m=4$ multipole perturbations and tidal shear, the time delay increases with ellipticity and shear, but is not very sensitive to $m=4$ modes.

Using Monte Carlo simulations, we then constructed distributions of the time delays in four-image lenses. This approach can handle fold, cusp, and cross lenses, which comprise the three canonical four-image lens morphologies. By constructing a catalog of mock lenses based on observed populations of elliptical galaxies, we computed the range of time delays and time delay ratios that would be expected for a smooth lens potential (i.e., one with ellipticity, shear, and $m=4$ multipoles). By comparing observed time delays with the predicted ranges, we have found time delay anomalies in the systems RX J0911+0551, RX J1131-1231, B1422+231, B1608+656, and WFI 2033-4723. It is unlikely that these anomalies can be explained by errors in our assumed values of the Hubble constant or the slope of the density profile: the Hubble constant would have to be unreasonably high or the density profile surprisingly shallow in order 
to explain some of the apparent anomalies (we specifically discussed RX J0911+0551 and WFI 2033-4723), and neither possibility could explain RX J1131-1231. It is possible to further reduce sensitivity to the Hubble constant and density profile by working with time delay ratios, although in general we have found that time delay ratios have less power to reveal anomalies. Part of the problem is that there are fewer time delay ratios known than time delays themselves, and a large uncertainty in a given time delay will lead to a correspondingly large uncertainty in the ratio, making definitive conclusions difficult.

In general, anomalies between close pairs of images in fold and cusp lenses should provide the cleanest evidence of substructure. The cusp lens RX J1131-1231 contains such anomalies, which is consistent with conclusions based on more detailed modeling by Morgan et al. $(2006)$ and Keeton \& Moustakas (2009). The cusp lenses B1422+231 and RX J0911+0551 show evidence of time delay anomalies in the cusp triplet, but the large uncertainties in the measured time delays prevent firm conclusions at present. The only fold pair that is clearly anomalous is in B1608+656, but the peculiar nature of this system (with two lens galaxies inside the Einstein angle) makes it difficult to draw a definitive conclusion about substructure from our analysis. Among largerseparation image pairs we have found time delay anomalies in the fold lens WFI 2033-4723 and the cusp lens RX J0911+0551. Both lenses show evidence of a complex environment, but in order to distinguish between that and substructure as the origin of the time delay anomalies it will be necessary to precisely measure the time delays between the close images (the fold pair in WFI 2033-4723, and the cusp triplet in RX J0911+0551).

In the hope that the sample of observed precision time delays will continue to grow, we have predicted the time delays for all mixed-parity image pairs in all 25 known four-image lenses. As new time delays are measured, it will be a simple matter to compare them with our predicted confidence intervals to determine whether they are anomalous. If a lens galaxy contains complex structure, time delays should help reveal it. Flux ratios provide a powerful way to find small-scale structure, but they are not unique in this; time delays hold great promise for contributing to our understanding of the role played by dark matter in the universe, especially when combined with other lensing observables.

We thank Leonidas Moustakas and Ross Fadely for helpful conversations. We also thank the referee, Olaf Wucknitz, for several helpful suggestions that improved the manuscript. ABC would also like to thank Mark Eichenlaub and Dennis Lam for their input. Part of this work was funded by NSF grant AST-0747311. ABC is currently supported by an appointment to the NASA Postdoctoral Program at the Jet Propulsion Laboratory, administered by Oak Ridge Associated Universities through a contract with NASA.

\section{REFERENCES}

Auger, M. W., Fassnacht, C. D., Abrahamse, A. L., Lubin, L. M., \& Squires, G. K. 2007, AJ, 134, $668[$ [ADS]

Barkana, R. 1997, ApJ, 489, 21 [ADS]

Bender, R., Surma, P., Döbereiner, S., Möllenhoff, C., \& Madejsky, R. 1989, A\&A, 217, 35 [ADS]

Bolton, A. S., Burles, S., Koopmans, L. V. E., Treu, T., Gavazzi, R., Moustakas, L. A., Wayth, R., \& Schlegel, D. J. 2008, ApJ, 682,964 [ADS]

Bolton, A. S., Burles, S., Koopmans, L. V. E., Treu, T., \& Moustakas, L. A. 2006, ApJ, 638, 703 [ADS]

Bradač, M., Schneider, P., Steinmetz, M., Lombardi, M., King, L. J., \& Porcas, R. 2002, A\&A, 388, 373 [ADS]

Burud, I., et al., 2002, A\&A, 383, 71 [ADS]

Chartas, G., Dai, X., Gallagher, S. C., Garmire, G. P., Bautz, M. W., Schechter, P. L., \& Morgan, N. D. 2001, ApJ, 558, 119 [ADS]

Chartas, G., Dai, X., \& Garmire, G. P. 2004, "Chandra and XMM-Newton Results on the Hubble Constant from Gravitational Lensing," in Measuring and Modeling the Universe, ed. W. L. Freedman (Pasadena: Carnegie Observatories) [ADS]

Chen, J., Rozo, E., Dalal, N., \& Taylor, J. E. 2007, ApJ, 659, 52 [ADS]

Chiba, M. 2002, ApJ, 565, 17 [ADS]

Chiba, M., Minezaki, T., Kashikawa, N., Kataza, H., \& Inoue, K. T. 2005, ApJ, 627, 53 [ADS]

Congdon, A. B., Keeton, C. R., \& Nordgren, C. E. 2008, MNRAS, 389, 398 [ADS]

Courbin, F. 2003, arXiv:astro-ph/0304497 [ADS]

Dalal, N., \& Kochanek, C. S. 2002, ApJ, 572, 25 [ADS]

Dobler, G., \& Keeton, C. R. 2006, MNRAS, 365, 1243 [ADS]

Eigenbrod, A., Courbin, F., Dye, S., Meylan, G., Sluse, D., Vuissoz, C., \& Magain, P. 2006, A\&A, 451, 747 [ADS]

Eigenbrod, A., Courbin, F., Sluse, D., Meylan, G., \& Agol, E. 2008, A\&A, 480, 647 [ADS]

Eigenbrod, A., Courbin, F., Vuissoz, C., Meylan, G., Saha, P., \& Dye, S. 2005, A\&A, 436, 25 [ADS]
Fassnacht, C. D., Xanthopoulos, E., Koopmans, L. V. E., \& Rusin, D. 2002, ApJ, 581, 823 [ADS]

Fohlmeister, J., Kochanek, C. S., Falco, E. E., Morgan, C. W., \& Wambsganss, J. 2008, ApJ, 676, 761 [ADS]

Hjorth, J., et al., 2002, ApJ, 572, L11 [ADS]

Holder, G. P., \& Schechter, P. L. 2003, ApJ, 589, 688 [ADS]

Inada, N., et al., 2005a, AJ, 130, 1967 [ADS]

Inada, N., et al., 2006, AJ, 131, 1934 [ADS]

Inada, N., et al., 2005b, PASJ, 57, L7 |ADS|

Inada, N., et al., 2003, Nature, 426, 810 [ADS]

Jørgensen, I., Franx, M., \& Kjærgaard, P. 1995, MNRAS, 273, 1097 [ADS]

Kayo, I., et al., 2007, AJ, 134, 1515 [ADS]

Keeton, C. R. 2001, arXiv:astro-ph/0102340 [ADS]

Keeton, C. R., Burles, S., Schechter, P. L., \& Wambsganss, J. 2006, ApJ, 639, 1 [ADS]

Keeton, C. R., Gaudi, B. S., \& Petters, A. O. 2003, ApJ, 598, 138 [ADS]

-. 2005, ApJ, 635, 35 [ADS]

Keeton, C. R., Kochanek, C. S., \& Seljak, U. 1997, ApJ, 482, 604 $[\mathrm{ADS}]$

Keeton, C. R., \& Moustakas, L. A. 2009, ApJ, 699, 1720 [ADS]

Keeton, C. R., \& Zabludoff, A. I. 2004, ApJ, 612, 660 [ADS]

Klypin, A., Kravtsov, A. V., Valenzuela, O., \& Prada, F. 1999, ApJ, 522, 82 [ADS]

Kneib, J.-P., Cohen, J. G., \& Hjorth, J. 2000, ApJ, 544, L35 $[\mathrm{ADS}]$

Kochanek, C. S. 2002, ApJ, 578, 25 [ADS]

Kochanek, C. S., Dai, X., Morgan, C., Morgan, N., \& Poindexter, S. C. G. 2007, in ASP Conf. Ser., Vol. 371, Statistical Challenges in Modern Astronomy IV, ed. G. J. Babu \& E. D. Feigelson, $43[$ [ADS]

Kochanek, C. S., \& Dalal, N. 2004, ApJ, 610, 69 [ADS]

Kochanek, C. S., Morgan, N. D., Falco, E. E., McLeod, B. A., Winn, J. N., Dembicky, J., \& Ketzeback, B. 2006a, ApJ, 640, $47[\mathrm{ADS}]$ 
Kochanek, C. S., Schneider, P., \& Wambsganss, J. 2006b, Saas-Fee Advanced Course 33: Gravitational Lensing: Strong, Weak and Micro, eds. G. Meylan, P. Jetzer, \& P. North (Heidelberg: Springer-Verlag) [ADS]

Koopmans, L. V. E., \& Fassnacht, C. D. 1999, ApJ, 527, 513 [ADS]

Koopmans, L. V. E., Treu, T., Bolton, A. S., Burles, S., \& Moustakas, L. A. 2006, ApJ, 649, 599 [ADS]

Koopmans, L. V. E., Treu, T., Fassnacht, C. D., Blandford, R. D., \& Surpi, G. 2003, ApJ, 599, 70 [ADS]

Mao, S., \& Schneider, P. 1998, MNRAS, 295, 587[ADDS]

Metcalf, R. B., \& Madau, P. 2001, ApJ, 563, 9 [ADS]

Metcalf, R. B., Moustakas, L. A., Bunker, A. J., \& Parry, I. R. 2004, ApJ, 607, 43 [ADS]

Metcalf, R. B., \& Zhao, H. 2002, ApJ, 567, L5 [ADS]

Minezaki, T., Chiba, M., Kashikawa, N., Inoue, K. T., \& Kataza, H. 2009, ApJ, 697, 610 [ADS]

Momcheva, I., Williams, K., Keeton, C., \& Zabludoff, A. 2006, ApJ, 641, 169 [ADS]

Moore, B., Ghigna, S., Governato, F., Lake, G., Quinn, T., Stadel, J., \& Tozzi, P. 1999, ApJ, 524, L19 [ADS]

Morgan, N. D., Caldwell, J. A. R., Schechter, P. L., Dressler, A., Egami, E., \& Rix, H.-W. 2004, AJ, 127, 2617 [ADS]

Morgan, N. D., Kochanek, C. S., Falco, E. E., \& Dai, X. 2006, arXiv:astro-ph/0605321 [ADS]

Morgan, N. D., Kochanek, C. S., Pevunova, O., \& Schechter, P. L. 2005, AJ, 129, 2531 [ADS]

Moustakas, L. A., et al. 2009, Astro 2010: The Astron. and Astroph. Decadal Survey, Science White Papers, no. 214 [ADS]

Moustakas, L. A., et al. 2008, Proc. SPIE, 7010, 70101B [ADS||

Moustakas, L. A., \& Metcalf, R. B. 2003, MNRAS, 339, 607 [ADS]

Navarro, J. F., Frenk, C. S., \& White, S. D. M. 1997, ApJ, 490, $493[$ [ADS]

Oguri, M. 2007, ApJ, 660, 1 [ADS]

Oguri, M., Inada, N., Blackburne, J. A., Shin, M.-S., Kayo, I., Strauss, M. A., Schneider, D. P., \& York, D. G. 2008, MNRAS, 391, 1973 [ADS]

Oguri, M., et al., 2004, ApJ, 605, 78 [ADS]

Patnaik, A. R., \& Narasimha, D. 2001, MNRAS, 326, 1403 [ADS]
Poindexter, S., Morgan, N., Kochanek, C. S., \& Falco, E. E. 2007a, ApJ, 667, 644 [ADS]

-. 2007b, ApJ, 660, 146 [ADS]

Pooley, D., Blackburne, J. A., Rappaport, S., \& Schechter, P. L. 2007, ApJ, 661, 19 [ADS]

Richards, et al., 2004, ApJ, 610, 679 [ADS]

Rusin, D., \& Kochanek, C. S. 2005, ApJ, 623, 666 [ADS]

Saglia, R. P., Bender, R., \& Dressler, A. 1993, A\&A, 279, 75 [ADS]

Saha, P., Courbin, F., Sluse, D., Dye, S., \& Meylan, G. 2006, A\&A, 450, $461[$ [ADS]

Saha, P., \& Williams, L. L. R. 2003, AJ, 125, 2769 [ADS]

Schechter, P. L., et al., 1997, ApJ, 475, L85 [ADS]

Schneider, P., Ehlers, J., \& Falco, E. E. 1992, Gravitational Lenses (Berlin: Springer-Verlag) [ADS]

Shakura, N. I., \& Sunyaev, R. A. 1973, A\&A, 24, 337 [ADS]

Sluse, D., Claeskens, J.-F., Hutsemékers, D., \& Surdej, J. 2007, A\&A, 468, $885[$ [ADS]

Sluse, D., Courbin, F', Eigenbrod, A., \& Meylan, G. 2008, A\&A, 492, L39[ADS]

Strigari, L. E., Bullock, J. S., Kaplinghat, M., Diemand, J., Kuhlen, M., \& Madau, P. 2007, ApJ, 669, 676 [ADS]

Suyu, S. H., Marshall, P. J., Blandford, R. D., Fassnacht, C. D., Koopmans, L. V. E., McKean, J. P., \& Treu, T. 2009, ApJ, $691,277[$ [ADS]

Treu, T., Koopmans, L. V., Bolton, A. S., Burles, S., \& Moustakas, L. A. 2006, ApJ, 640, 662 [ADS]

Treu, T., \& Koopmans, L. V. E. 2004, ApJ, 611, 739 [ADS]

Vuissoz, C., et al., 2008, A\&A, 488, 481[ADS]

Warren, S. J., Hewett, P. C., Lewis, G. F.,, Moller, P., Iovino, A., \& Shaver, P. A. 1996, MNRAS, 278, 139 [ADS]

Warren, S. J., Lewis, G. F., Hewett, P. C., Møller, P., Shaver, P., \& Iovino, A. 1999, A\&A, 343, L35 [ADS]

Wisotzki, L., Becker, T., Christensen, L., Helms, A., Jahnke, K., Kelz, A., Roth, M. M., \& Sanchez, S. F. 2003, A\&A, 408, 455 [ADS]

Woźniak, P. R., Alard, C., Udalski, A., Szymański, M., Kubiak, M., Pietrzyński, G., \& Zebruń, K. 2000, ApJ, 529, 88 [ADS] 

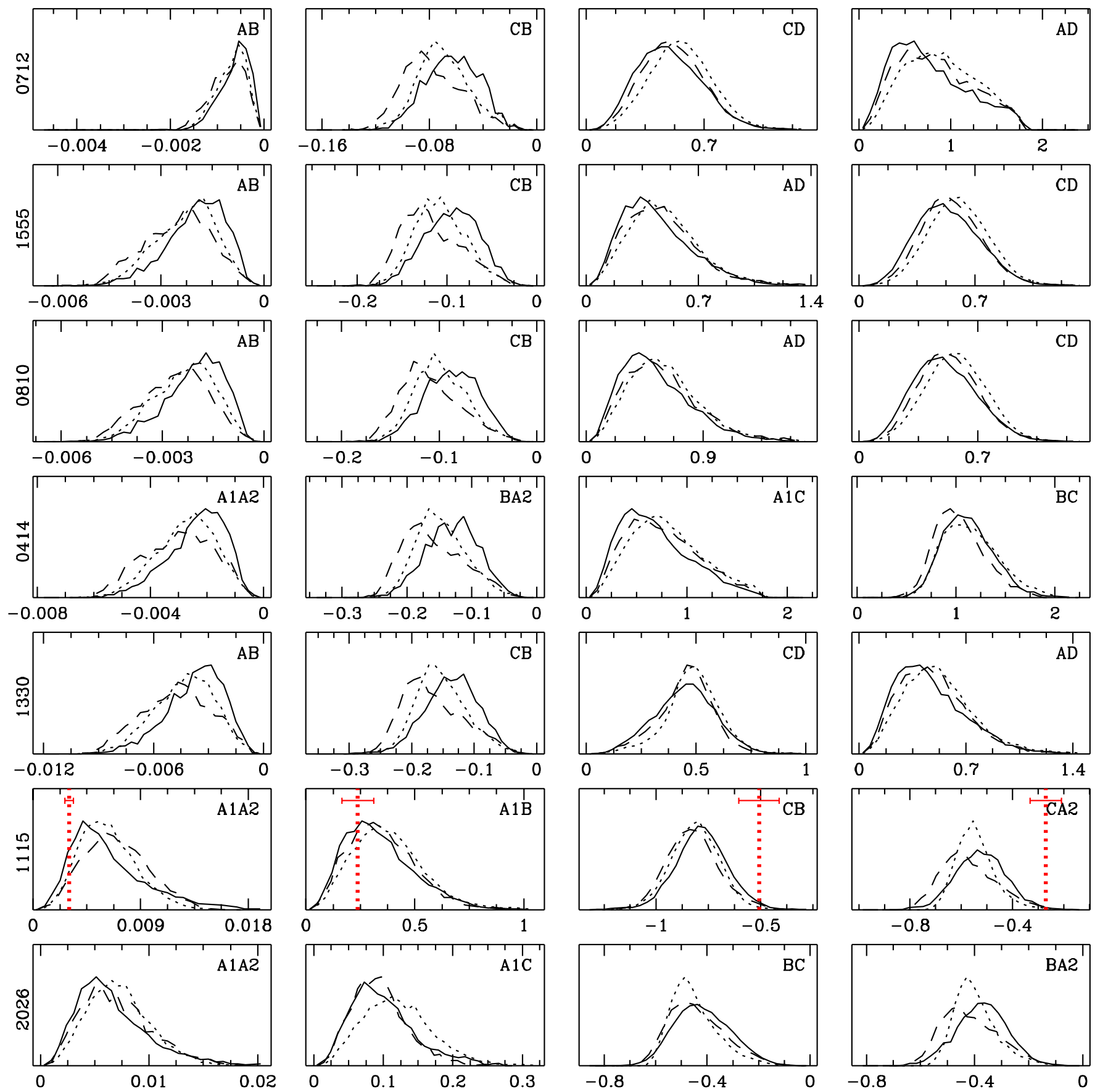

FIG. 3. - Time delay histograms for the known fold lenses. The horizontal axes show the scaled time delay in units of $\tau_{0} \theta_{E}^{2}$; the range is chosen to span three standard deviations above and below the mean of the predicted time-delay distribution, and further expanded if necessary to encompass the observed value. The vertical axes are in arbitrary units, with each panel scaled to the maximum value of its three histograms. From top to bottom, lenses are arranged in order of increasing $d_{1}^{*}$; the abbreviated lens name appears at the far left. (See Table 1 for the full names.) From left to right, the panels correspond to image pairs with increasing values of $d_{1}$. The solid, dotted, and dashed curves show histograms corresponding to the data of Bender et al. (1989), Jørgensen et al. (1995), and Saglia et al. (1993), respectively. For image pairs with observed time delays, vertical dashed lines show the measured values. The errorbars show $3 \sigma$ measurement uncertainties (see Table2). In cases where measurement uncertainty formally allows for time delays whose signs are disallowed by the parity cut (see $\$ 3$ ), errorbars are truncated at vanishing abscissa. 

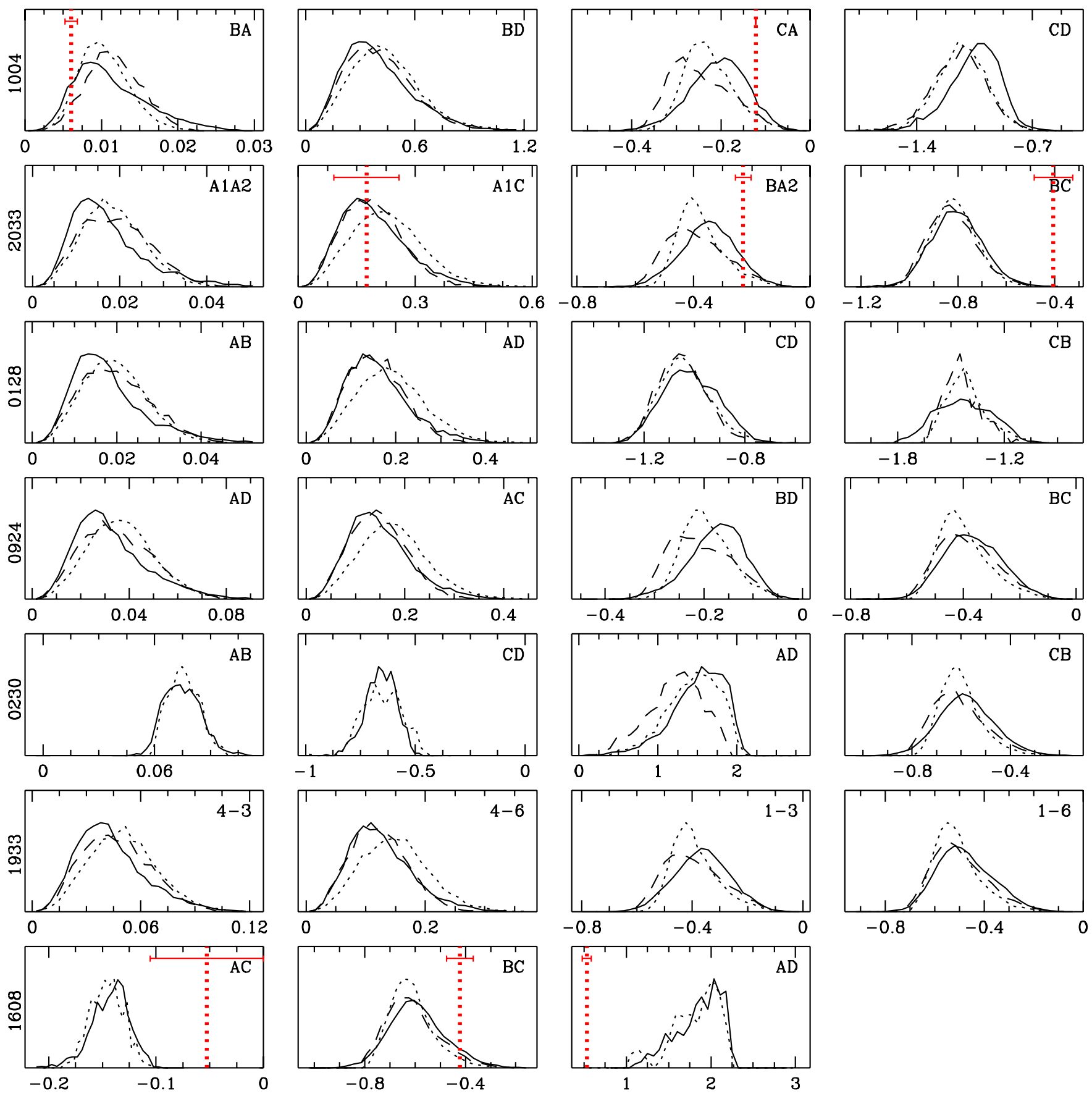

FIG. 4. - Time delay histograms for fold lenses (continued). 

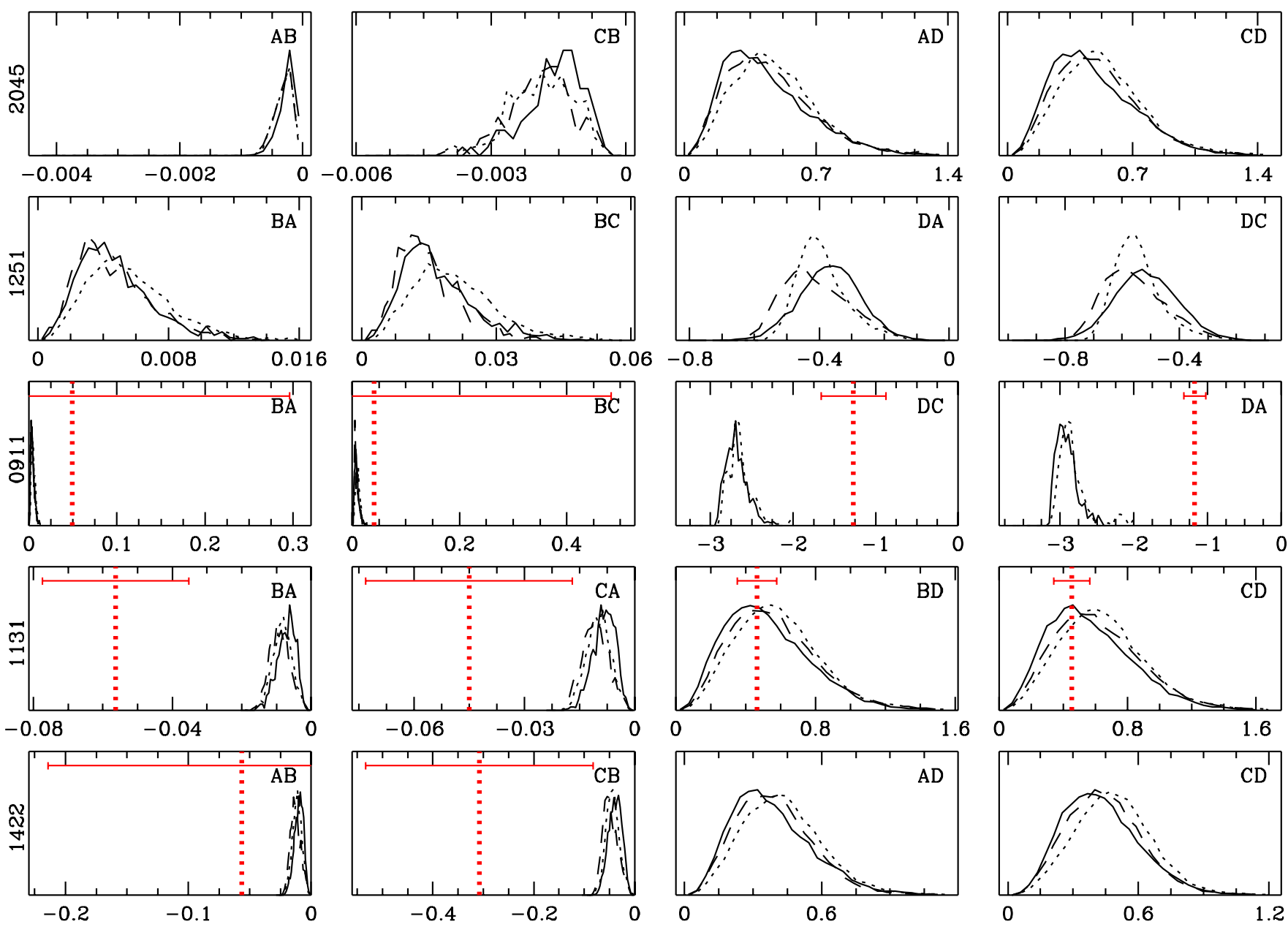

FIg. 5.- Same as Figure 3 but for the known cusp lenses. 
Identifying Anomalies in Gravitational Lens Time Delays
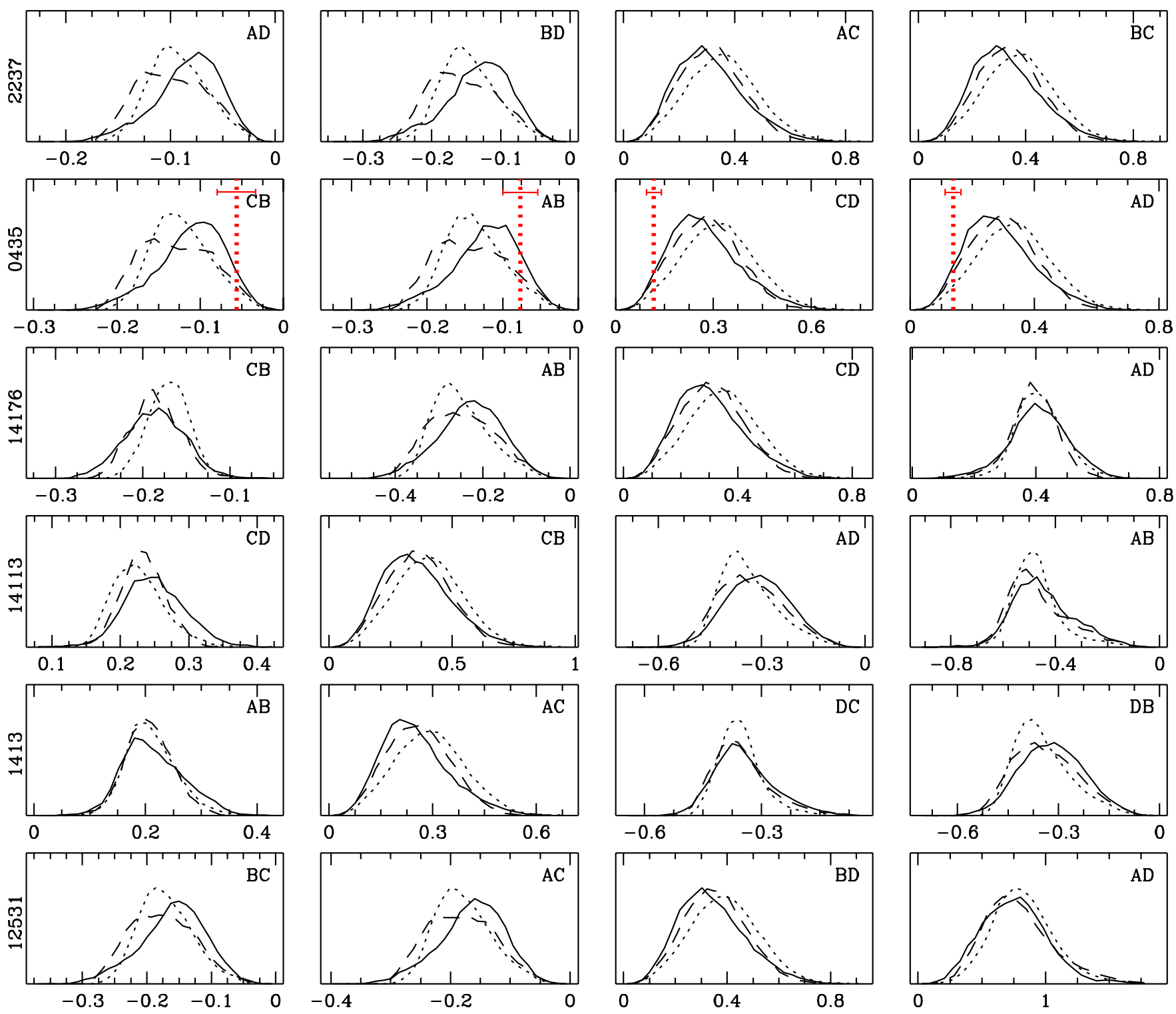

FIG. 6.- Same as Figure 3 but for the known cross lenses. 

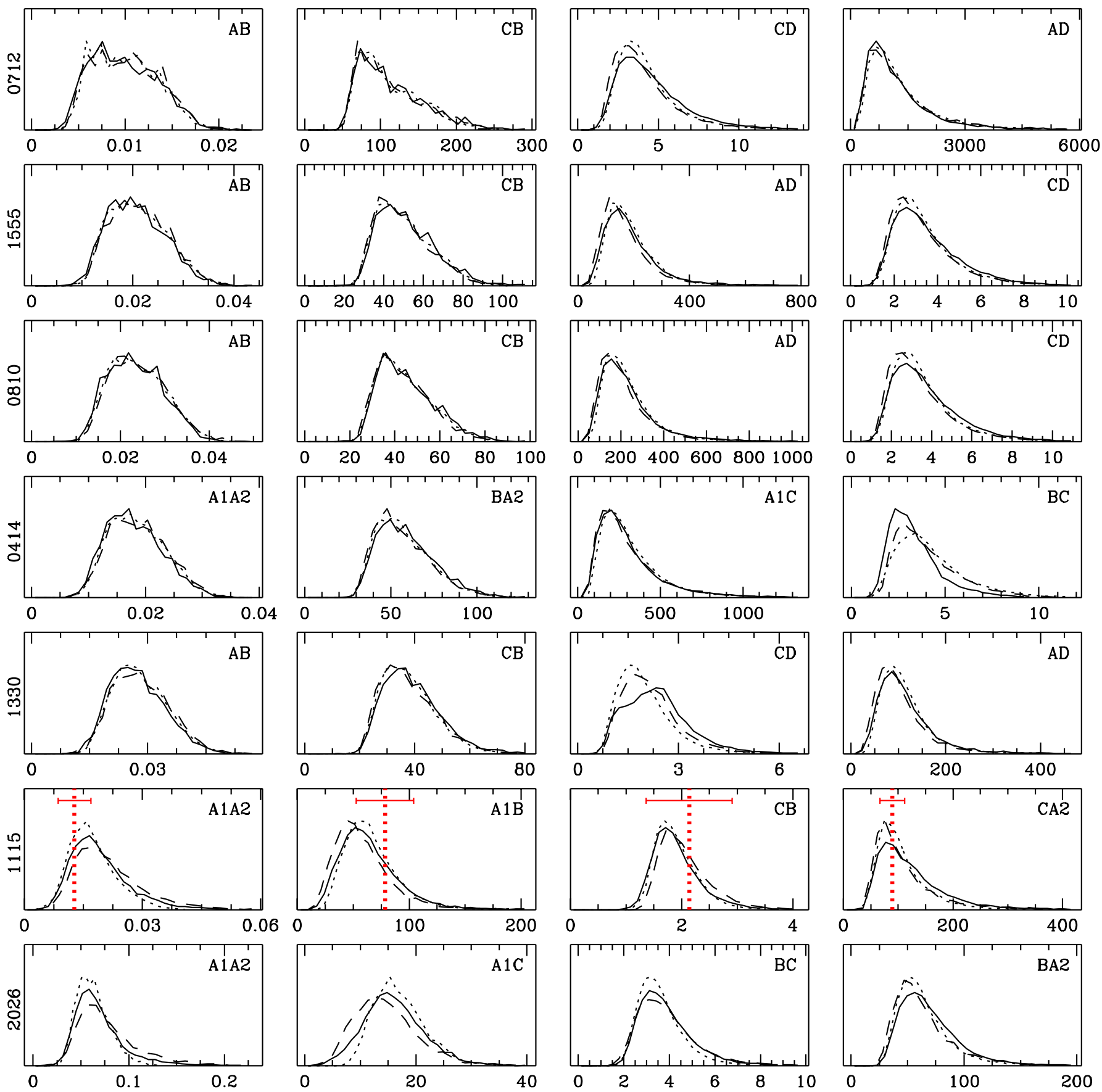

Fig. 7. - Histograms of time delay ratios for the known fold lenses. The horizontal axes show the (dimensionless) time delay ratio. The vertical axes are in arbitrary units, with each panel scaled to the maximum value of its three histograms. From top to bottom, lenses are arranged in order of increasing $d_{1}^{*}$; the abbreviated lens name appears at far left. (See Table 1 for the full names.) From left to right, the panels correspond to image pairs with increasing values of $d_{1}$. The solid, dotted, and dashed curves show histograms corresponding to the data of Bender et al. (1989), Jørgensen et al. (1995), and Saglia et al. (1993), respectively. For image pairs where it is possible to construct time delay ratios from observational data, vertical dashed lines show these values. The errorbars show $3 \sigma$ measurement uncertainties (see

Table 3). Truncated errorbars have the same meaning as in Figure 3 

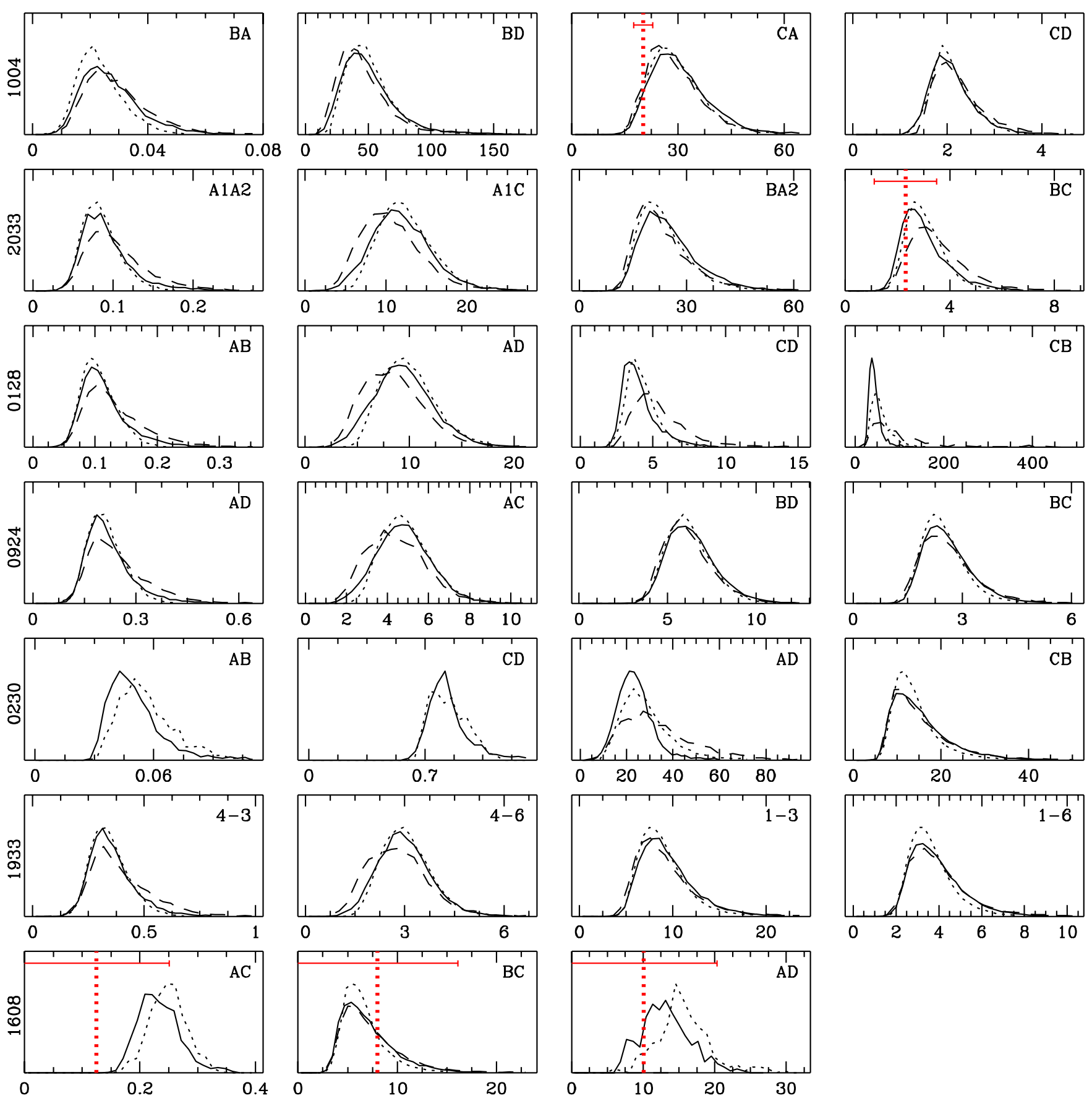

FIG. 8.- Histograms of time delay ratios for fold lenses (continued).

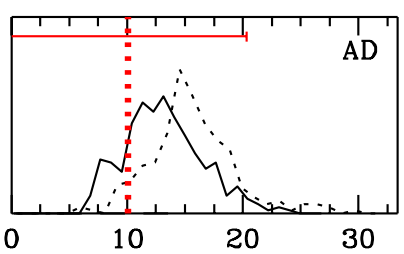



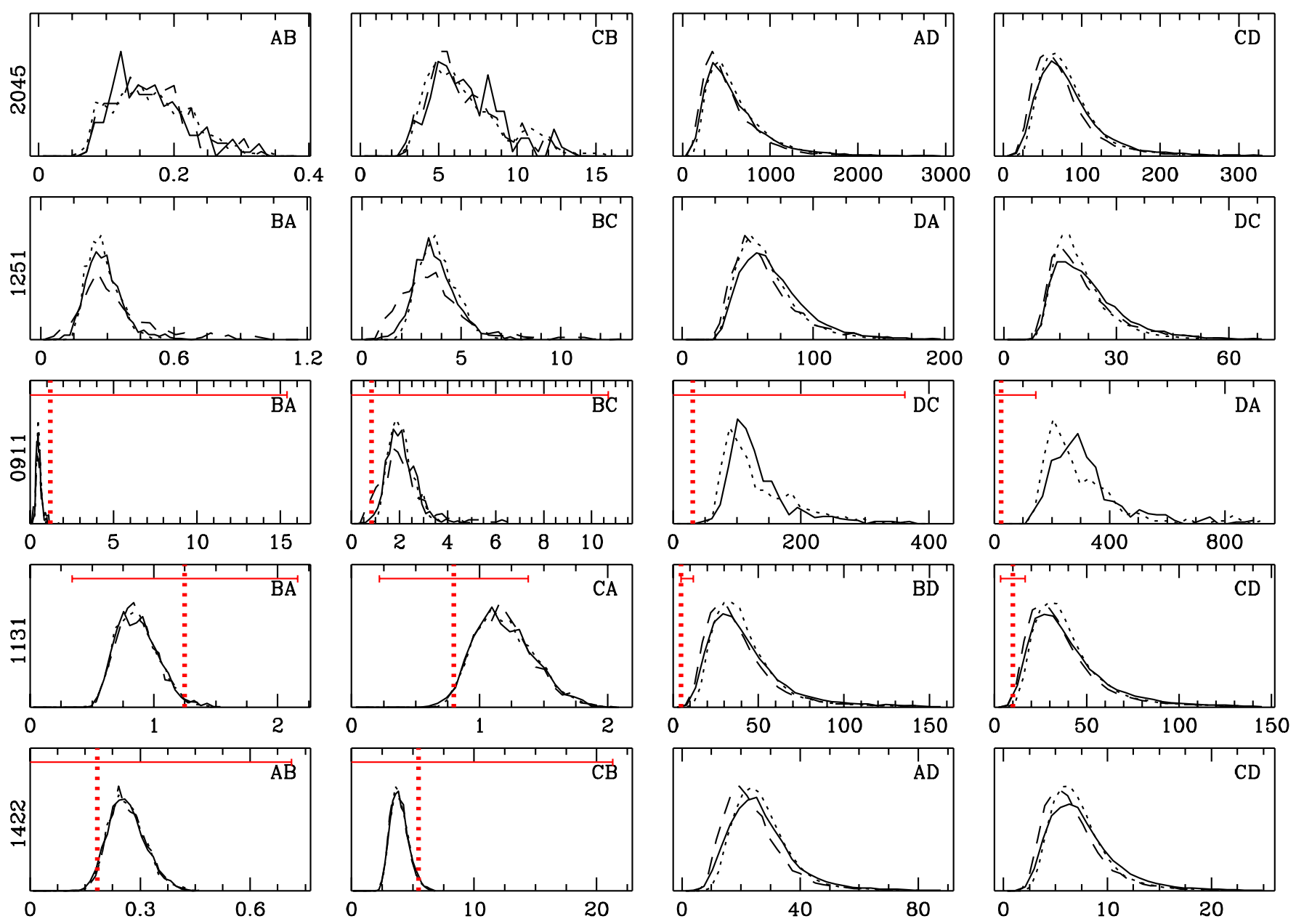

FIG. 9.- Same as Figure 7 but for the known cusp lenses. 

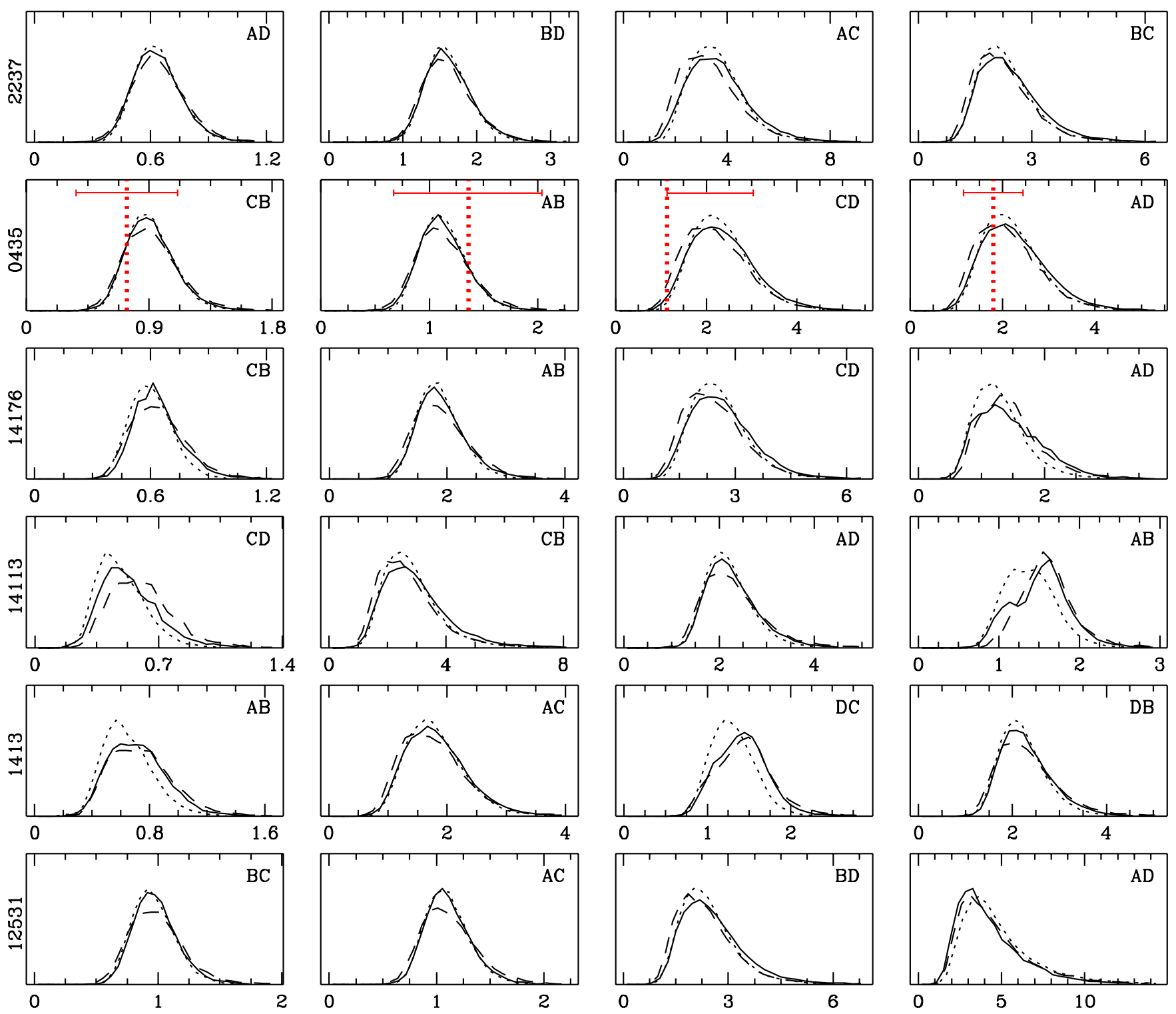

FIG. 10.- Same as Figure 7 but for the known cross lenses.
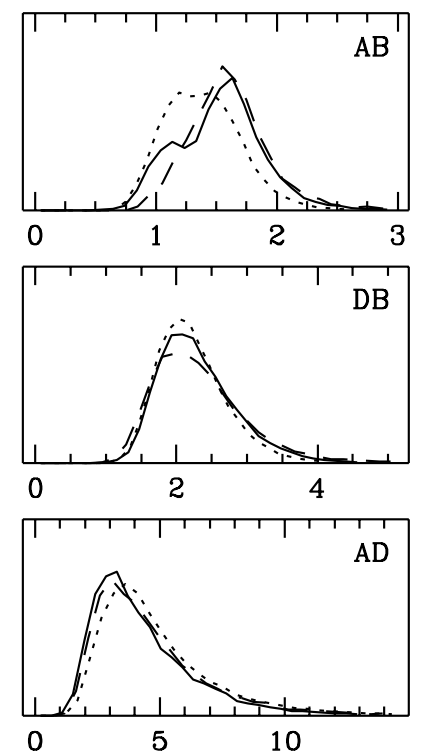
TABLE 4

MEDian VALUES AND CONFIDENCE INTERVALS FOR SCALED TIME DELAYS

\begin{tabular}{|c|c|c|c|c|c|}
\hline $\begin{array}{l}\text { Lens } \\
\text { Name }\end{array}$ & $\begin{array}{l}\text { Image } \\
\text { Pair }\end{array}$ & $\begin{array}{l}d_{1} \\
(\operatorname{arcsec})\end{array}$ & $\begin{array}{r}\text { Median } \\
\Delta t_{1}\end{array}$ & $\begin{array}{l}\text { 95\% Conf. } \\
\text { Interval }\end{array}$ & $\begin{array}{l}99 \% \text { Conf. } \\
\text { Interval }\end{array}$ \\
\hline 0128 & $\mathrm{AB}[\mathrm{D}]$ & 0.14 & 0.0164 & $(0.00598,0.0403)$ & $(0.00386,0.0483)$ \\
\hline 0128 & $\mathrm{AD}[\mathrm{B}]$ & 0.27 & 0.152 & $(0.0527,0.332)$ & $(0.0333,0.394)$ \\
\hline 0128 & $\mathrm{CD}[\mathrm{A}]$ & 0.42 & -1.02 & $(-1.22,-0.800)$ & $(-1.26,-0.716)$ \\
\hline 0128 & $\mathrm{CB}[\mathrm{A}]$ & 0.5 & -1.43 & $(-1.72,-1.14)$ & $(-1.78,-1.03)$ \\
\hline 0230 & $\mathrm{AB}[\mathrm{D}]$ & 0.74 & 0.0745 & $(0.0583,0.0954)$ & $(0.0547,0.104)$ \\
\hline 0230 & $\mathrm{CD}[\mathrm{A}]$ & 1.46 & -0.654 & $(-0.806,-0.537)$ & $(-0.848,-0.507)$ \\
\hline 0230 & $\mathrm{AD}[\mathrm{B}]$ & 1.64 & 1.56 & $(0.728,1.99)$ & $(0.436,2.05)$ \\
\hline 0230 & $\mathrm{CB}[\mathrm{A}]$ & 1.65 & -0.583 & $(-0.792,-0.349)$ & $(-0.858,-0.271)$ \\
\hline 0414 & $\mathrm{~A} 1 \mathrm{~A} 2[\mathrm{~B}]$ & 0.41 & -0.00223 & $(-0.00471,-0.000827)$ & $(-0.00553,-0.000520)$ \\
\hline 0414 & BA2[A1] & 1.71 & -0.129 & $(-0.212,-0.0560)$ & $(-0.248,-0.0383)$ \\
\hline 0414 & $\mathrm{~A} 1 \mathrm{C}[\mathrm{A} 2]$ & 1.96 & 0.635 & $(0.178,1.50)$ & $(0.112,1.66)$ \\
\hline 0414 & $\mathrm{BC}[\mathrm{A} 2]$ & 2.13 & 1.10 & $(0.668,1.69)$ & $(0.536,1.97)$ \\
\hline 0712 & $\mathrm{AB}[\mathrm{C}]$ & 0.17 & -0.000571 & $(-0.00136,-0.000185)$ & $(-0.00161,-0.000148)$ \\
\hline 0712 & $\mathrm{CB}[\mathrm{A}]$ & 0.91 & -0.0627 & $(-0.101,-0.0272)$ & $(-0.113,-0.0203)$ \\
\hline 0712 & $\mathrm{CD}[\mathrm{B}]$ & 1.18 & 0.484 & $(0.177,0.911)$ & $(0.118,1.12)$ \\
\hline 0712 & $\mathrm{AD}[\mathrm{B}]$ & 1.25 & 0.697 & $(0.177,1.66)$ & $(0.105,1.77)$ \\
\hline 0810 & $\mathrm{AB}[\mathrm{C}]$ & 0.18 & -0.00195 & $(-0.00407,-0.000712)$ & $(-0.00474,-0.000503)$ \\
\hline 0810 & $\mathrm{CB}[\mathrm{A}]$ & 0.69 & -0.0868 & $(-0.144,-0.0377)$ & $(-0.160,-0.0274)$ \\
\hline 0810 & $\mathrm{AD}[\mathrm{B}]$ & 0.84 & 0.494 & $(0.147,1.19)$ & $(0.0950,1.46)$ \\
\hline 0810 & $\mathrm{CD}[\mathrm{B}]$ & 0.85 & 0.498 & $(0.188,0.920)$ & $(0.128,1.13)$ \\
\hline 0924 & $\mathrm{AD}[\mathrm{C}]$ & 0.69 & 0.0297 & $(0.0108,0.0688)$ & $(0.00737,0.0816)$ \\
\hline 0924 & $\mathrm{AC}[\mathrm{D}]$ & 1.18 & 0.141 & $(0.0494,0.303)$ & $(0.0332,0.353)$ \\
\hline 0924 & $\mathrm{BD}[\mathrm{A}]$ & 1.46 & -0.170 & $(-0.298,-0.0738)$ & $(-0.334,-0.0506)$ \\
\hline 0924 & $\mathrm{BC}[\mathrm{A}]$ & 1.53 & -0.370 & $(-0.549,-0.160)$ & $(-0.597,-0.108)$ \\
\hline 1004 & $\mathrm{BA}[\mathrm{D}]$ & 3.73 & 0.0104 & $(0.00424,0.0225)$ & $(0.00307,0.0254)$ \\
\hline 1004 & $\mathrm{BD}[\mathrm{A}]$ & 11.44 & 0.367 & $(0.115,0.839)$ & $(0.0748,1.10)$ \\
\hline 1004 & $\mathrm{CA}[\mathrm{B}]$ & 11.84 & -0.203 & $(-0.344,-0.0912)$ & $(-0.383,-0.0629)$ \\
\hline 1004 & $\mathrm{CD}[\mathrm{B}]$ & 14.38 & -1.04 & $(-1.40,-0.797)$ & $(-1.55,-0.698)$ \\
\hline 1115 & $\mathrm{~A} 1 \mathrm{~A} 2[\mathrm{~B}]$ & 0.48 & 0.00559 & $(0.00195,0.0147)$ & $(0.00136,0.0170)$ \\
\hline 1115 & $\mathrm{~A} 1 \mathrm{~B}[\mathrm{~A} 2]$ & 1.67 & 0.316 & $(0.0995,0.730)$ & $(0.0657,0.976)$ \\
\hline 1115 & $\mathrm{CB}[\mathrm{A} 1]$ & 1.99 & -0.783 & $(-1.04,-0.529)$ & $(-1.19,-0.437)$ \\
\hline 1115 & CA2[A1] & 2.16 & -0.525 & $(-0.716,-0.325)$ & $(-0.794,-0.249)$ \\
\hline 1330 & $\mathrm{AB}[\mathrm{C}]$ & 0.43 & -0.00355 & $(-0.00739,-0.00129)$ & $(-0.00867,-0.000872)$ \\
\hline 1330 & $\mathrm{CB}[\mathrm{A}]$ & 1.53 & -0.135 & $(-0.222,-0.0595)$ & $(-0.259,-0.0419)$ \\
\hline 1330 & $\mathrm{CD}[\mathrm{B}]$ & 1.64 & 0.454 & $(0.175,0.718)$ & $(0.117,0.823)$ \\
\hline 1330 & $\mathrm{AD}[\mathrm{B}]$ & 1.65 & 0.428 & $(0.130,1.01)$ & $(0.0815,1.26)$ \\
\hline 1555 & $\mathrm{AB}[\mathrm{C}]$ & 0.09 & -0.00188 & $(-0.00397,-0.000676)$ & $(-0.00451,-0.000449)$ \\
\hline 1555 & $\mathrm{CB}[\mathrm{A}]$ & 0.35 & -0.0933 & $(-0.155,-0.0410)$ & $(-0.171,-0.0301)$ \\
\hline 1555 & $\mathrm{AD}[\mathrm{B}]$ & 0.4 & 0.406 & $(0.124,0.975)$ & $(0.0785,1.23)$ \\
\hline 1555 & $\mathrm{CD}[\mathrm{B}]$ & 0.42 & 0.525 & $(0.204,0.951)$ & $(0.135,1.17)$ \\
\hline 1608 & $\mathrm{AC}[\mathrm{B}]$ & 0.87 & -0.142 & $(-0.184,-0.111)$ & $(-0.202,-0.106)$ \\
\hline 1608 & $\mathrm{BC}[\mathrm{A}]$ & 1.51 & -0.598 & $(-0.789,-0.351)$ & $(-0.856,-0.267)$ \\
\hline 1608 & $\mathrm{AD}[\mathrm{C}]$ & 1.69 & 1.91 & $(1.23,2.21)$ & $(1.10,2.27)$ \\
\hline 1608 & $\mathrm{BD}[\mathrm{C}]$ & 2.00 & 1.19 & - & - \\
\hline 1933 & 4_3[6] & 0.46 & 0.0407 & $(0.0144,0.0888)$ & $(0.00911,0.104)$ \\
\hline 1933 & $4 \_6[3]$ & 0.63 & 0.119 & $(0.0413,0.252)$ & $(0.0271,0.294)$ \\
\hline 1933 & $1 \_3[4]$ & 0.9 & -0.364 & $(-0.559,-0.171)$ & $(-0.618,-0.120)$ \\
\hline 1933 & $1 \_6[4]$ & 0.91 & -0.488 & $(-0.672,-0.238)$ & $(-0.732,-0.167)$ \\
\hline 2026 & $\mathrm{~A} 1 \mathrm{~A} 2[\mathrm{C}]$ & 0.33 & 0.00601 & $(0.00198,0.0154)$ & $(0.00137,0.0198)$ \\
\hline 2026 & $\mathrm{~A} 1 \mathrm{C}[\mathrm{A} 2]$ & 0.83 & 0.0929 & $(0.0308,0.218)$ & $(0.0200,0.258)$ \\
\hline 2026 & $\mathrm{BC}[\mathrm{A} 1]$ & 1.19 & -0.424 & $(-0.609,-0.195)$ & $(-0.664,-0.135)$ \\
\hline 2026 & $\mathrm{BA} 2[\mathrm{~A} 1]$ & 1.28 & -0.372 & $(-0.561,-0.188)$ & $(-0.624,-0.127)$ \\
\hline 2033 & $\mathrm{~A} 1 \mathrm{~A} 2[\mathrm{C}]$ & 0.72 & 0.0155 & $(0.00572,0.0392)$ & $(0.00372,0.0469)$ \\
\hline 2033 & $\mathrm{~A} 1 \mathrm{C}[\mathrm{A} 2]$ & 1.54 & 0.182 & $(0.0623,0.392)$ & $(0.0421,0.463)$ \\
\hline 2033 & $\mathrm{BA} 2[\mathrm{~A} 1]$ & 2.01 & -0.348 & $(-0.537,-0.172)$ & $(-0.586,-0.116)$ \\
\hline 2033 & $\mathrm{BC}[\mathrm{A} 1]$ & 2.13 & -0.806 & $(-1.00,-0.579)$ & $(-1.07,-0.494)$ \\
\hline 0911 & $\mathrm{BA}[\mathrm{C}]-\mathrm{A} 2 \mathrm{~A} 1[\mathrm{~A} 3]$ & 0.48 & 0.00401 & $(0.00128,0.0104)$ & $(0.000726,0.0134)$ \\
\hline 0911 & $\mathrm{BC}[\mathrm{A}]-\mathrm{A} 2 \mathrm{~A} 3[\mathrm{~A} 1]$ & 0.62 & 0.00776 & $(0.00243,0.0208)$ & $(0.00178,0.0281)$ \\
\hline 0911 & $\mathrm{DC}[\mathrm{B}]-\mathrm{BA} 3[\mathrm{~A} 2]$ & 2.96 & -2.70 & $(-2.88,-2.38)$ & $(-2.90,-2.24)$ \\
\hline 0911 & $\mathrm{DA}[\mathrm{B}]-\mathrm{BA} 1[\mathrm{~A} 2]$ & 3.08 & -2.93 & $(-3.11,-2.56)$ & $(-3.12,-2.47)$ \\
\hline 1131 & $\mathrm{BA}[\mathrm{C}]$ & 1.19 & -0.00680 & $(-0.0130,-0.00271)$ & $(-0.0150,-0.00185)$ \\
\hline 1131 & $\mathrm{CA}[\mathrm{B}]$ & 1.26 & -0.00813 & $(-0.0148,-0.00331)$ & $(-0.0171,-0.00230)$ \\
\hline 1131 & $\mathrm{BD}[\mathrm{A}]$ & 3.14 & 0.492 & $(0.152,1.09)$ & $(0.0971,1.33)$ \\
\hline 1131 & $\mathrm{CD}[\mathrm{A}]$ & 3.18 & 0.533 & $(0.162,1.17)$ & $(0.0991,1.40)$ \\
\hline 1251 & $\mathrm{BA}[\mathrm{C}]$ & 0.44 & 0.00435 & $(0.00139,0.0109)$ & $(0.000879,0.0131)$ \\
\hline 1251 & $\mathrm{BC}[\mathrm{A}]$ & 0.7 & 0.0153 & $(0.00510,0.0388)$ & $(0.00339,0.0532)$ \\
\hline 1251 & $\mathrm{DA}[\mathrm{B}]$ & 1.72 & -0.366 & $(-0.553,-0.182)$ & $(-0.619,-0.122)$ \\
\hline 1251 & $\mathrm{DC}[\mathrm{B}]$ & 1.77 & -0.519 & $(-0.728,-0.291)$ & $(-0.802,-0.220)$ \\
\hline 1422 & $\mathrm{AB}[\mathrm{C}]$ & 0.5 & -0.00981 & $(-0.0181,-0.00374)$ & $(-0.0204,-0.00261)$ \\
\hline 1422 & $\mathrm{CB}[\mathrm{A}]$ & 0.82 & -0.0381 & $(-0.0655,-0.0153)$ & $(-0.0726,-0.0103)$ \\
\hline 1422 & $\mathrm{AD}[\mathrm{B}]$ & 1.25 & 0.360 & $(0.118,0.808)$ & $(0.0767,1.07)$ \\
\hline 1422 & $\mathrm{CD}[\mathrm{B}]$ & 1.29 & 0.413 & $(0.145,0.830)$ & $(0.0947,1.05)$ \\
\hline 2045 & $\mathrm{AB}[\mathrm{C}]$ & 0.28 & -0.000236 & $(-0.000611,-0.0000811)$ & $(-0.000745,-0.0000684)$ \\
\hline
\end{tabular}


Identifying Anomalies in Gravitational Lens Time Delays

TABLE 4

Median VALUes AND CONFIDENCE INTERVALS FOR SCALEd TIME DELAYS

\begin{tabular}{|c|c|c|c|c|c|}
\hline 2045 & $\mathrm{CB}[\mathrm{A}]$ & 0.56 & -0.00151 & $(-0.00294,-0.000679)$ & $(-0.00339,-0.000554)$ \\
\hline 2045 & $\mathrm{AD}[\mathrm{B}]$ & 1.91 & 0.398 & $(0.112,1.00)$ & $(0.0693,1.24)$ \\
\hline 2045 & $\mathrm{CD}[\mathrm{B}]$ & 1.93 & 0.443 & $(0.134,1.02)$ & $(0.0804,1.26)$ \\
\hline 0435 & $\mathrm{CB}[\mathrm{A}]$ & 1.53 & -0.107 & $(-0.202,-0.0437)$ & $(-0.227,-0.0289)$ \\
\hline 0435 & $\mathrm{AB}[\mathrm{C}]$ & 1.59 & -0.119 & $(-0.224,-0.0484)$ & $(-0.251,-0.0329)$ \\
\hline 0435 & $\mathrm{CD}[\mathrm{B}]$ & 1.85 & 0.261 & $(0.0997,0.519)$ & $(0.0667,0.598)$ \\
\hline 0435 & $\mathrm{AD}[\mathrm{B}]$ & 1.88 & 0.277 & $(0.106,0.544)$ & $(0.0718,0.623)$ \\
\hline 12531 & $\mathrm{BC}[\mathrm{A}]$ & 0.77 & -0.156 & $(-0.269,-0.0696)$ & $(-0.297,-0.0466)$ \\
\hline 12531 & $\mathrm{AC}[\mathrm{B}]$ & 0.78 & -0.160 & $(-0.283,-0.0688)$ & $(-0.318,-0.0460)$ \\
\hline 12531 & $\mathrm{BD}[\mathrm{C}]$ & 0.91 & 0.330 & $(0.128,0.634)$ & $(0.0856,0.726)$ \\
\hline 12531 & $\mathrm{AD}[\mathrm{C}]$ & 1.02 & 0.749 & $(0.297,1.30)$ & $(0.201,1.58)$ \\
\hline 14113 & $\mathrm{CD}[\mathrm{B}]$ & 1.13 & 0.248 & $(0.169,0.341)$ & $(0.135,0.376)$ \\
\hline 14113 & $\mathrm{CB}[\mathrm{D}]$ & 1.38 & 0.344 & $(0.129,0.661)$ & $(0.0869,0.762)$ \\
\hline 14113 & $\mathrm{AD}[\mathrm{C}]$ & 1.41 & -0.311 & $(-0.486,-0.133)$ & $(-0.538,-0.0898)$ \\
\hline 14113 & $\mathrm{AB}[\mathrm{C}]$ & 1.42 & -0.461 & $(-0.641,-0.192)$ & $(-0.729,-0.133)$ \\
\hline 1413 & $\mathrm{AB}[\mathrm{C}]$ & 0.76 & 0.208 & $(0.118,0.330)$ & $(0.0870,0.361)$ \\
\hline 1413 & $\mathrm{AC}[\mathrm{B}]$ & 0.87 & 0.239 & $(0.0941,0.478)$ & $(0.0620,0.547)$ \\
\hline 1413 & $\mathrm{DC}[\mathrm{A}]$ & 0.91 & -0.354 & $(-0.473,-0.165)$ & $(-0.509,-0.107)$ \\
\hline 1413 & $\mathrm{DB}[\mathrm{A}]$ & 0.96 & -0.320 & $(-0.498,-0.135)$ & $(-0.547,-0.0924)$ \\
\hline 14176 & $\mathrm{CB}[\mathrm{A}]$ & 1.73 & -0.186 & $(-0.254,-0.119)$ & $(-0.273,-0.0913)$ \\
\hline 14176 & $\mathrm{AB}[\mathrm{C}]$ & 2.09 & -0.226 & $(-0.382,-0.0955)$ & $(-0.426,-0.0649)$ \\
\hline 14176 & $\mathrm{CD}[\mathrm{B}]$ & 2.13 & 0.292 & $(0.109,0.573)$ & $(0.0753,0.661)$ \\
\hline 14176 & $\mathrm{AD}[\mathrm{B}]$ & 2.13 & 0.409 & $(0.200,0.597)$ & $(0.126,0.649)$ \\
\hline 2237 & $\mathrm{AD}[\mathrm{B}]$ & 1.01 & -0.0801 & $(-0.155,-0.0318)$ & $(-0.174,-0.0220)$ \\
\hline 2237 & $\mathrm{BD}[\mathrm{A}]$ & 1.18 & -0.128 & $(-0.239,-0.0527)$ & $(-0.264,-0.0369)$ \\
\hline 2237 & $\mathrm{AC}[\mathrm{D}]$ & 1.37 & 0.297 & $(0.108,0.586)$ & $(0.0729,0.682)$ \\
\hline 2237 & $\mathrm{BC}[\mathrm{D}]$ & 1.4 & 0.316 & $(0.122,0.607)$ & $(0.0820,0.704)$ \\
\hline
\end{tabular}

Note. - The first column gives the abbreviated lens name (the full names appear in the first column of Table 1). The next two columns list the image pair label and the separation between the images in arcseconds. The letter in brackets in column two indicates the third image needed to compute time delay ratios. The last three columns present data computed from our numerical simulations, using the galaxy sample of Bender et al. (1989). The fourth column gives the median value of the differential time delay in units of $\tau_{0} \theta_{E}^{2}$ (cf. Table 2), and the fifth and sixth columns give the $95 \%$ and $99 \%$ confidence intervals of this same quantity.

TABLE 5

MEdian VAlues AND CONFIDENCE INTERVALS FOR TIME DELAY RATIOS

\begin{tabular}{lllrcc}
\hline \hline $\begin{array}{l}\text { Lens } \\
\text { Name }\end{array}$ & Image & $\begin{array}{l}d_{1} \\
(\operatorname{arcsec})\end{array}$ & $\begin{array}{r}\text { Median } \\
\Delta t_{1} / \Delta t_{2}\end{array}$ & $\begin{array}{c}95 \% \text { Conf. } \\
\text { Interval }\end{array}$ & $\begin{array}{c}99 \% \text { Conf. } \\
\text { Interval }\end{array}$ \\
\hline 0128 & AB & 0.14 & 0.107 & $(0.0631,0.230)$ & $(0.0499,0.334)$ \\
0128 & AD & 0.27 & 9.31 & $(4.34,15.9)$ & $(2.99,20.0)$ \\
0128 & CD & 0.42 & 3.82 & $(2.48,7.73)$ & $(2.13,10.4)$ \\
0128 & CB & 0.5 & 41.7 & $(27.1,101)$. & $(25.2,160)$. \\
0230 & AB & 0.74 & 0.0477 & $(0.0330,0.0942)$ & $(0.0310,0.124)$ \\
0230 & CD & 1.46 & 0.818 & $(0.667,1.17)$ & $(0.633,1.29)$ \\
0230 & AD & 1.64 & 22.9 & $(11.3,47.5)$ & $(8.58,70.0)$ \\
0230 & CB & 1.65 & 13.9 & $(7.41,33.7)$ & $(6.46,46.0)$ \\
0414 & A1A2 & 0.41 & 0.0175 & $(0.00996,0.0288)$ & $(0.00794,0.0318)$ \\
0414 & BA2 & 1.71 & 57.2 & $(34.6,100)$. & $(31.4,126)$. \\
0414 & A1C & 1.96 & 261. & $(92.7,997)$. & $(70.2,1650)$. \\
0414 & BC & 2.13 & 3.08 & $(1.57,7.38)$ & $(1.25,9.64)$ \\
0712 & AB & 0.17 & 0.00961 & $(0.00448,0.0170)$ & $(0.00359,0.0193)$ \\
0712 & CB & 0.91 & 104. & $(58.4,220)$. & $(50.9,269)$. \\
0712 & CD & 1.18 & 4.12 & $(1.83,10.7)$ & $(1.43,14.0)$ \\
0712 & AD & 1.25 & 996. & $(310 ., 4170)$. & $(215 ., 6910)$. \\
0810 & AB & 0.18 & 0.0230 & $(0.0132,0.0360)$ & $(0.0111,0.0408)$ \\
0810 & CB & 0.69 & 43.5 & $(27.7,75.7)$ & $(24.4,89.9)$ \\
0810 & AD & 0.84 & 205. & $(76.9,761)$. & $(57.6,1370)$ \\
0810 & CD & 0.85 & 3.38 & $(1.59,8.43)$ & $(1.27,11.1)$ \\
0924 & AD & 0.69 & 0.210 & $(0.128,0.422)$ & $(0.105,0.564)$ \\
0924 & AC & 1.18 & 4.77 & $(2.37,7.84)$ & $(1.77,9.52)$ \\
0924 & BD & 1.46 & 6.22 & $(4.12,9.90)$ & $(3.56,12.0)$ \\
0924 & BC & 1.53 & 2.50 & $(1.61,4.41)$ & $(1.40,5.45)$ \\
1004 & BA & 3.73 & 0.0256 & $(0.0123,0.0533)$ & $(0.00918,0.0683)$ \\
1004 & BD & 11.44 & 46.8 & $(20.8,121)$. & $(16.3,213)$. \\
1004 & CA & 11.84 & 29.4 & $(18.3,51.5)$ & $(15.7,63.0)$ \\
1004 & CD & 14.38 & 2.03 & $(1.38,3.30)$ & $(1.23,4.03)$ \\
1115 & A1A2 & 0.48 & 0.0179 & $(0.00849,0.0397)$ & $(0.00650,0.0514)$ \\
1115 & A1B & 1.67 & 60.2 & $(26.4,141)$. & $(20.1,232)$. \\
1115 & CB & 1.99 & 1.83 & $(1.28,3.08)$ & $(1.12,3.83)$ \\
1115 & CA2 & 2.16 & 108. & $(51.1,323)$. & $(44.2,494)$. \\
1330 & AB & 0.43 & 0.0267 & $(0.0155,0.0426)$ & $(0.0135,0.0474)$ \\
\hline & & & & &
\end{tabular}


TABLE 5

Median Values AND CONFIDENCE INTERVAls For time DELAy RATios

\begin{tabular}{|c|c|c|c|c|c|}
\hline 1330 & $\mathrm{CB}$ & 1.53 & 37.5 & $(23.5,64.4)$ & $(21.0,74.2)$ \\
\hline 1330 & CD & 1.64 & 2.29 & $(0.937,4.89)$ & $(0.766,6.72)$ \\
\hline 1330 & $\mathrm{AD}$ & 1.65 & 102. & $(41.8,323 .)^{\prime}$ & $(30.5,591)$. \\
\hline 1555 & $\mathrm{AB}$ & 0.09 & 0.0202 & $(0.0116,0.0327)$ & $(0.00928,0.0361)$ \\
\hline 1555 & $\mathrm{CB}$ & 0.35 & 49.4 & $(30.6,86.2)$ & $(27.6,107)$. \\
\hline 1555 & $\mathrm{AD}$ & 0.4 & 168. & $(66.3,553)$. & $(51.7,1030)$. \\
\hline 1555 & $\mathrm{CD}$ & 0.42 & 3.21 & $(1.52,7.86)$ & $(1.21,10.2)$ \\
\hline 1608 & $\mathrm{AC}$ & 0.87 & 0.229 & $(0.175,0.320)$ & $(0.162,0.351)$ \\
\hline 1608 & $\mathrm{BC}$ & 1.51 & 6.48 & $(3.69,14.3)$ & $(3.20,19.0)$ \\
\hline 1608 & $\mathrm{AD}$ & 1.69 & 13.1 & $(7.29,20.8)$ & $(6.72,23.2)$ \\
\hline 1608 & $\mathrm{BD}$ & 2.00 & 2.21 & - & \\
\hline 1933 & 4_3 & 0.46 & 0.340 & $(0.207,0.638)$ & $(0.166,0.829)$ \\
\hline 1933 & 4_6 & 0.63 & 2.94 & $(1.57,4.83)$ & $(1.20,5.99)$ \\
\hline 1933 & 1_3 & 0.9 & 8.93 & $(5.41,17.4)$ & $(4.67,22.6)$ \\
\hline 1933 & $1 \_6$ & 0.91 & 3.67 & $(2.21,7.20)$ & $(1.89,9.26)$ \\
\hline 2026 & A1A2 & 0.33 & 0.0640 & $(0.0350,0.156)$ & $(0.0280,0.230)$ \\
\hline 2026 & $\mathrm{~A} 1 \mathrm{C}$ & 0.83 & 15.6 & $(6.41,28.5)$ & $(4.27,35.4)$ \\
\hline 2026 & $\mathrm{BC}$ & 1.19 & 3.64 & $(2.22,6.94)$ & $(1.89,8.77)$ \\
\hline 2026 & BA2 & 1.28 & 64.0 & $(34.4,143)$. & $(29.8,208)$. \\
\hline 2033 & $\mathrm{~A} 1 \mathrm{~A} 2$ & 0.72 & 0.0856 & $(0.0484,0.187)$ & $(0.0384,0.247)$ \\
\hline 2033 & $\mathrm{~A} 1 \mathrm{C}$ & 1.54 & 11.7 & $(5.34,20.7)$ & $(4.04,26.0)$ \\
\hline 2033 & BA2 & 2.01 & 23.9 & $(13.9,47.8)$ & $(12.2,65.7)$ \\
\hline 2033 & $\mathrm{BC}$ & 2.13 & 2.86 & $(1.84,5.49)$ & $(1.60,6.88)$ \\
\hline 0911 & BA & 0.48 & 0.495 & $(0.262,0.956)$ & $(0.171,1.23)$ \\
\hline 0911 & $\mathrm{BC}$ & 0.62 & 2.01 & $(1.03,3.70)$ & $(0.754,5.73)$ \\
\hline 0911 & $\mathrm{DC}$ & 2.96 & 118. & $(73.6,292)$. & $(55.1,551)$. \\
\hline 0911 & DA & 3.08 & 292. & $(156 ., 741)$. & $(126 ., 912)$. \\
\hline 1131 & BA & 1.19 & 0.856 & $(0.589,1.25)$ & $(0.548,1.42)$ \\
\hline 1131 & $\mathrm{CA}$ & 1.26 & 1.17 & $(0.800,1.70)$ & $(0.696,1.82)$ \\
\hline 1131 & $\mathrm{BD}$ & 3.14 & 37.5 & $(15.2,120)$. & $(11.4,199)$. \\
\hline 1131 & $\mathrm{CD}$ & 3.18 & 35.2 & $(14.1,112)$. & $(10.5,170)$. \\
\hline 1251 & BA & 0.44 & 0.278 & $(0.148,0.558)$ & $(0.114,0.824)$ \\
\hline 1251 & $\mathrm{BC}$ & 0.7 & 3.59 & $(1.79,6.73)$ & $(1.18,8.75)$ \\
\hline 1251 & DA & 1.72 & 64.9 & $(34.8,147)$. & $(30.4,211)$. \\
\hline 1251 & DC & 1.77 & 20.7 & $(11.0,50.3)$ & $(9.43,68.9)$ \\
\hline 1422 & $\mathrm{AB}$ & 0.5 & 0.261 & $(0.177,0.376)$ & $(0.157,0.412)$ \\
\hline 1422 & $\mathrm{CB}$ & 0.82 & 3.83 & $(2.65,5.64)$ & $(2.42,6.31)$ \\
\hline 1422 & $\mathrm{AD}$ & 1.25 & 25.3 & $(11.1,62.4)$ & $(8.60,106)$. \\
\hline 1422 & $\mathrm{CD}$ & 1.29 & 7.02 & $(3.13,18.1)$ & $(2.48,29.7)$ \\
\hline 2045 & $\mathrm{AB}$ & 0.28 & 0.155 & $(0.0809,0.293)$ & $(0.0735,0.323)$ \\
\hline 2045 & $\mathrm{CB}$ & 0.56 & 6.34 & $(3.29,12.2)$ & $(3.02,12.8)$ \\
\hline 2045 & $\mathrm{AD}$ & 1.91 & 508. & (185., 1890.) & $(130 ., 3580)$. \\
\hline 2045 & $\mathrm{CD}$ & 1.93 & 75.6 & $(30.4,234)$. & $(23.1,406)$. \\
\hline 0435 & $\mathrm{CB}$ & 1.53 & 0.899 & $(0.604,1.34)$ & $(0.502,1.62)$ \\
\hline 0435 & $\mathrm{AB}$ & 1.59 & 1.11 & $(0.744,1.66)$ & $(0.618,2.00)$ \\
\hline 0435 & $\mathrm{CD}$ & 1.85 & 2.30 & $(1.23,4.08)$ & $(0.997,5.02)$ \\
\hline 0435 & $\mathrm{AD}$ & 1.88 & 2.19 & $(1.18,3.99)$ & $(0.982,5.03)$ \\
\hline 12531 & $\mathrm{BC}$ & 0.77 & 0.967 & $(0.670,1.42)$ & $(0.587,1.69)$ \\
\hline 12531 & $\mathrm{AC}$ & 0.78 & 1.09 & $(0.754,1.63)$ & $(0.638,1.99)$ \\
\hline 12531 & $\mathrm{BD}$ & 0.91 & 2.42 & $(1.28,5.12)$ & $(1.04,7.47)$ \\
\hline 12531 & $\mathrm{AD}$ & 1.02 & 3.83 & $(1.80,10.0)$ & $(1.44,13.4)$ \\
\hline 14113 & CD & 1.13 & 0.515 & $(0.285,0.968)$ & $(0.221,1.23)$ \\
\hline 14113 & CB & 1.38 & 2.79 & $(1.41,6.07)$ & $(1.15,8.95)$ \\
\hline 14113 & $\mathrm{AD}$ & 1.41 & 2.19 & $(1.45,3.63)$ & $(1.25,4.41)$ \\
\hline 14113 & $\mathrm{AB}$ & 1.42 & 1.54 & $(0.887,2.27)$ & $(0.777,2.64)$ \\
\hline 1413 & $\mathrm{AB}$ & 0.76 & 0.702 & $(0.389,1.18)$ & $(0.314,1.50)$ \\
\hline 1413 & $\mathrm{AC}$ & 0.87 & 1.77 & $(1.00,3.08)$ & $(0.832,3.77)$ \\
\hline 1413 & $\mathrm{DC}$ & 0.91 & 1.41 & $(0.896,2.07)$ & $(0.790,2.47)$ \\
\hline 1413 & DB & 0.96 & 2.21 & $(1.46,3.67)$ & $(1.26,4.47)$ \\
\hline 14176 & CB & 1.73 & 0.625 & $(0.431,0.959)$ & $(0.366,1.17)$ \\
\hline 14176 & $\mathrm{AB}$ & 2.09 & 1.86 & $(1.23,2.95)$ & $(1.04,3.56)$ \\
\hline 14176 & $\mathrm{CD}$ & 2.13 & 2.56 & $(1.34,4.82)$ & $(1.08,6.27)$ \\
\hline 14176 & $\mathrm{AD}$ & 2.13 & 1.37 & $(0.693,2.75)$ & $(0.564,3.65)$ \\
\hline 2237 & $\mathrm{AD}$ & 1.01 & 0.627 & $(0.417,0.922)$ & $(0.350,1.07)$ \\
\hline 2237 & $\mathrm{BD}$ & 1.18 & 1.60 & $(1.09,2.41)$ & $(0.935,2.88)$ \\
\hline 2237 & $\mathrm{AC}$ & 1.37 & 3.55 & $(1.80,6.69)$ & $(1.45,8.74)$ \\
\hline 2237 & $\mathrm{BC}$ & 1.4 & 2.31 & $(1.25,4.72)$ & $(1.02,6.70)$ \\
\hline
\end{tabular}

Note. - The first three columns are the same as those in Table 4. The last three columns present data computed from our numerical simulations, using the galaxy sample of Bender et al. (1989). The fourth column gives the median value of the time delay ratio $\Delta t_{1} / \Delta t_{2}$. The subscripts on $\Delta t$ refer to the time delay of the labeled image pair $\left(\Delta t_{1}\right)$ and that for the closest neighboring pair $\left(\Delta t_{2}\right)$. The fifth and sixth columns give the $95 \%$ and $99 \%$ confidence intervals of the time delay ratio. 